\title{
Noise Reduction in Coarse Bifurcation Analysis of Stochastic Agent-Based Models: An Example of Consumer Lock-In*
}

\author{
Daniele Avitabile ${ }^{\dagger}$ Rebecca Hoyle ${ }^{\ddagger}$, and Giovanni Samaey§
}

\begin{abstract}
We investigate coarse equilibrium states of a fine-scale, stochastic, agent-based model of consumer lock-in in a duopolistic market. In the model, agents decide on their next purchase based on a combination of their personal preference and their neighbors' opinions. For agents with independent identically distributed (i.i.d.) parameters and all-to-all coupling, we derive an analytic approximate coarse evolution-map for the expected average purchase. We then study the emergence of coarse fronts when the agents are split into two factions with opposite preferences. We develop a novel Newton-Krylov method that is able to compute accurately and efficiently coarse fixed points when the underlying fine-scale dynamics is stochastic. The main novelty of the algorithm is in the elimination of the noise that is generated when estimating Jacobian-vector products using time-integration of perturbed initial conditions. We present numerical results that demonstrate the convergence properties of the numerical method and use the method to show that macroscopic fronts in this model destabilize at a coarse symmetry-breaking bifurcation.
\end{abstract}

Key words. equation-free methods, agent-based models, multiple-scale analysis

AMS subject classifications. 37M02, 65P30, 91C02

DOI. $10.1137 / 140962188$

1. Introduction. Understanding how social groups reach general agreement or perform a coordinated task has been the subject of an intense research effort over the past 50 years [10]. In models of social behavior, consensus is a macroscopic feature emerging from random reciprocal interactions between a large number of heterogeneous actors. Understanding how consensus arises and identifying the key factors for its generation or inhibition are fundamental questions in social dynamics.

A large class of social models, known as sociophysical models [23], is based on an analogy with ferromagnetism: social attributes such as opinions or preferences then correspond to magnetic dipole moments of atomic spins, and choices are influenced by interactions with neighboring spins; consensus is then represented by a phase transition $[9,12,57]$ that is studied with the tools of statistical mechanics. Sociophysical models have been applied in various

\footnotetext{
${ }^{*}$ Received by the editors March 24, 2014; accepted for publication (in revised form) by J. Sieber September 3, 2014; published electronically November 18, 2014.

http://www.siam.org/journals/siads/13-4/96218.html

†'School of Mathematical Sciences, University of Nottingham, Nottingham, NG2 7RD, UK (daniele.avitabile@nottingham.ac.uk). The work of this author was supported by the Engineering and Physical Sciences Research Council (EPSRC) and was partially supported by EPSRC grant EP/H021779/1.

${ }^{\ddagger}$ Department of Mathematics, University of Surrey, Guildford, Surrey GU2 7XH, UK (r.hoyle@surrey.ac.uk). The work of this author was partially supported by EPSRC grant EP/H021779/1.

${ }^{\S}$ Department of Computer Science, K. U. Leuven, Celestijnenlaan 200A, 3001 Leuven, Belgium (giovanni.samaey@cs.kuleaven.be). The work of this author was partially supported by the Research Council of KU Leuven through grant OT/13/66, by the Interuniversity Attraction Poles Programme of the Belgian Science Policy Office under grant IUAP/V/22, and by the Research Foundation-Flanders (FWO) through grant G.A003.13.
} 1583

Copyright ( by SIAM. Unauthorized reproduction of this article is prohibited. 
social contexts to study, for instance, segregation (Ising and Schelling models) [52, 25, 58], opinion formation [34,60], and social impact [43]. We refer the reader to the reviews by Schweitzer [53], Castellano, Fortunato, and Loreto [10], and Chakrabarti, Chakraborti, and Chatterjee [11] for further examples.

In statistical mechanical models, macroscopic coherent states emerge from the interaction of a large number of identical particles whose behavior obeys well-known physical principles. However, particle-like descriptions of social actors may be seen as simplistic, as, in social systems, individuals do not behave according to precise physical laws: collective behavior is the result of the interaction between complex heterogeneous entities which often make unpredictable decisions. An alternative strategy is to use agent-based models (ABMs) [20, 4, 27]. ABMs provide a bottom-up approach to social modeling in that they focus directly on individual actors. In ABMs, modelers prescribe detailed rules for agents' behavior, possibly including heterogeneities, stochasticity, memory effects, and bounded rationality. Agents exchange information with each other and influence (and are influenced by) their environment, which may be a model of a physical space or a network. Because of these characteristics, ABMs have become a popular tool in social sciences, with applications including crowd dynamics [33, 49], civil violence [19], urban crime [54], opinion dynamics [18, 31, 44, 45], and social networks [48]. They have also been used to model biological systems [30, 28, 62, 6]. In addition, several ABM libraries and software packages are available (see [50] for a review).

Even though ABMs allow a great level of granularity, it is often interesting to extract macroscopic variables from the system, study their asymptotic behavior, and explore their dependence upon control parameters. Sociodynamical models, pioneered by Weidlich [65, 66], are obtained by choosing appropriate coarse variables for the system under consideration and deriving master equations for the time evolution of their probability distributions; assuming that the distributions are unimodal and sharply peaked, an approximate closed nonlinear model for the first few distribution moments is then derived and analyzed with tools from dynamical systems theory. For a detailed review of techniques and applications of sociodynamics, we refer the reader to a recent book by Helbing [32]. In general, however, the induced closure approximations may be either insufficiently accurate or (in more complicated situations) impossible to perform, resulting in evolution equations for coarse variables that are hard or impossible to derive. In those cases, parameter variations are typically explored via brute-force Monte Carlo simulations, which give access only to stable asymptotic states and may require long transient simulations [59, 16, 1, 61].

The past decade has seen a growing interest in the development and deployment of computational methods that aim at accelerating multiple-scale simulations using on-the-fly numerical closure approximations. We mention here equation-free [37, 38] and heterogeneous multiscale methods [17,67]. Equation-free methods, in particular, are an effective bridge between the microscopic descriptions of sociophysical models or ABMs and the macroscopic viewpoint of sociodynamical models, since they not only allow for accelerated simulation at the macroscopic level but also enable system-level tasks, such as macroscopic bifurcation analysis. In the equation-free framework [37, 38], one assumes the existence of a closed macroscopic model in terms of a few macroscopic state variables. However, instead of deriving an approximate macroscopic model analytically, one constructs a computational superstructure, wrapped around a microscopic simulation. In this context, a key tool is the coarse time-

Copyright (c) by SIAM. Unauthorized reproduction of this article is prohibited. 
stepper, which implements a time step of a macroscopic model that is not available in closed form as a three-step procedure: (i) lifting, that is, the creation of initial conditions for the microscopic model, conditioned upon the macroscopic state at a given time $t$; (ii) simulation, using the microscopic model over a time interval $[t, t+T]$; and (iii) restriction, that is, the estimation of the macroscopic state at $t+T$.

While equation-free methods have been employed in various contexts $[13,22,41,21,40$, $39,42,63,56,14,35,47]$, several numerical issues remain, mainly related to the stochastic nature of the microscopic evolution. In the present paper, we focus on some of these numerical aspects while performing a coarse-grained bifurcation analysis of a stochastic ABM for opinion formation. In particular, the computation of macroscopic steady states requires the solution of a nonlinear system of algebraic equations, which is usually carried out via Newton-Krylov solvers built around the coarse time-stepper. If the underlying microscopic evolution equation is stochastic, numerical noise can severely affect Jacobian evaluations, representing a serious obstacle to the convergence of the nonlinear iterations [35].

The present paper deals with the numerical computation of macroscopic coherent structures for a model of vendor lock-in. Lock-in is achieved when customers repeatedly purchase the same product, irrespective of its quality, because choosing an alternative vendor is inconvenient or impossible. The term was originally used to explain the emergence of technological standards, with classic examples being the prevalence of VHS over Betamax videocassette recorders and of QWERTY over Dvorak layouts for computer keyboards [15, 2, 3, 36]. The starting point of our investigation is an ABM of vendor lock-in for duopolistic markets [24]: Garlick and Chli proposed this model in order to study, via direct numerical simulations, how to break lock-in. We extend their model so as to include stochastic dynamics and heterogeneities in the agents' preferences and perform a coarse numerical bifurcation analysis of two types of macroscopic steady states: a global locked-in state, where the entire agent population polarizes homogeneously, and fronts, which arise when two factions of agents have conflicting preferences.

The present paper thus contains two main contributions. First, for the specific system under study, we explain the birth of the above-described macroscopic states in terms of coarse symmetry-breaking bifurcations. To the best of our knowledge, steps in this direction were taken only very recently $[55,7]$ and were confined to globally locked-in states. In the homogeneous case, we follow [5] and interpret metastable locked-in states as fixed points of a coarse evolution map. In the limit of infinitely many globally coupled agents with homogeneous product preferences, we derive the coarse evolution map analytically. In the case of heterogeneous agents, we employ stochastic continuation and show for the first time how fronts destabilize to partially locked-in states.

The second main contribution of the paper is the development of a novel procedure to obtain coarse Jacobian-vector products with reduced variance, allowing the accurate evaluation of Jacobian-vector products in the presence of microscopic stochasticity, thus gaining full control over the linear and the nonlinear iterations of the Newton-Krylov solver. Even though our implementation of variance-reduced Jacobian-vector products is specific to the lock-in model, we believe that analogous strategies can be applied to other ABMs. Therefore, we provide a detailed account of the algorithmic steps involved in defining an accurate equation-free Newton-Krylov method and testing its convergence properties.

Copyright (c) by SIAM. Unauthorized reproduction of this article is prohibited. 
The paper is organized as follows: section 2 contains the description of the lock-in model and a preliminary simulation-based study of coarse macroscopic states; in section 3 we derive an approximate analytic coarse map for the case of homogeneous agents; in section 4 we describe the macroscopic time-stepper for the lock-in model and introduce weighted lifting operators to obtain variance-reduced Jacobian-vector products; in section 5 we test numerical properties of the Newton-GMRES solver; in section 6 we present the results of the coarse bifurcation analysis, and we conclude in section 7 .

\section{An ABM for consumer lock-in.}

2.1. Model description. In this section, we introduce a generalization of a consumer lock-in ABM proposed by Garlick and Chli [24], which, in our investigation, will serve as a prototypical ABM with heterogeneous agents and binary state variables.

Let us consider a set of $N$ agents on a two-dimensional square lattice spanning $[-1,1]^{2}$, in which the agents are placed on evenly spaced points $\left(x_{i}, y_{j}\right)_{i, j=1,1}^{I, J}$, with $x_{i}=-1+i \Delta x$ and $y_{j}=-1+j \Delta y$, such that $x_{I}=y_{J}=1$ and $I J=N$. For notational convenience, we use a lexicographic numbering of the agents, which are identified by a single index $n$ running from 1 to $N$. The position of the $n$th agent on the lattice is then denoted by $\boldsymbol{r}_{n}=\left(x_{n}, y_{n}\right)=\left(x_{i}, y_{j}\right)$ with $i=n \bmod I$ and $n=(j-1) I+i$.

At each discrete time step $t$, agents choose simultaneously between two products, labeled 0 and 1 , so that the associated state variables $u_{n}(t)$ are collected in a vector $\boldsymbol{u}(t) \in \mathbb{B}^{N}$, where $\mathbb{B}=\{0,1\}$. Agents are coupled via their neighborhoods $\square_{n}$, comprising $\left|\square_{n}\right|$ other agents, and their choices are determined by two parameters: the perceived relative quality $q_{n}$ of both products and each agent's tendency to follow its neighborhood, $\lambda_{n}$. If $q_{n} \approx-1$, then the $n$th agent has an intrinsic preference for product 0 over product 1 (and the opposite is true if $q_{n} \approx 1$ ). On the other hand, a value $\lambda_{n} \approx 0$ indicates that the $n$th agent disregards the opinion of its neighbors, whereas $\lambda_{n} \approx 1$ implies that the agent aligns itself with the majority of the neighbors. While these parameters remain constant at all times, each agent draws its values from an approximate normal distribution, whose moments may depend upon the position $\boldsymbol{r}_{n}$,

$$
q_{n} \sim \mathcal{N}\left(q ; \mu\left(\boldsymbol{r}_{n}\right), \xi\left(\boldsymbol{r}_{n}\right)\right), \quad \lambda_{n} \sim \mathcal{N}\left(\lambda ; \nu\left(\boldsymbol{r}_{n}\right), \zeta\left(\boldsymbol{r}_{n}\right)\right), \quad n=1, \ldots, N .
$$

In practice we require some constraints on $q_{n}$ and $\lambda_{n}$, namely, $q_{n} \in[-1,1]$ and $\lambda_{n} \in[0,1]$ : the normal distributions are chosen such that this occurs with very high probability; otherwise, the values of $q_{n}$ and $\lambda_{n}$ are discarded, and a new random value is generated. We point out that the issue of negative parameter values could also be avoided by prescribing distributions which are naturally defined on finite intervals (for instance, the Beta distribution); we have chosen the distributions as in the original model by Garlick and Chli [24].

Agent diversity is therefore modeled in two ways: $q_{n}$ and $\lambda_{n}$ are randomly generated, and the corresponding probability distributions may vary along the lattice. In this paper, we will choose

$$
\begin{array}{ll}
\mu\left(\boldsymbol{r}_{n}\right):=\mu\left(x_{n}\right)=\bar{\mu}+\Delta \mu \tanh \left(\alpha x_{n}\right), \quad & \xi\left(\boldsymbol{r}_{n}\right)=\bar{\xi}, \\
\nu\left(\boldsymbol{r}_{n}\right)=\bar{\nu}, & \zeta\left(\boldsymbol{r}_{n}\right)=\bar{\zeta}
\end{array}
$$

for $n=1, \ldots, N, \bar{\mu}, \alpha \in \mathbb{R}$, and $\Delta \mu, \bar{\xi}, \bar{\nu}, \bar{\zeta} \in \mathbb{R}^{+}$. Note that with the above choice, we only introduce a one-dimensional parametrization of the mean preferences. More general multi-

Copyright (c) by SIAM. Unauthorized reproduction of this article is prohibited. 
dimensional parametrizations of preferences and agents' tendency to follow their neighborhood are conceivable but will not be considered in this paper. As we shall see in the following sections, the sigmoid $\mu\left(x_{n}\right)$ allows us to model the existence of factions with strong preferences for one product.

At each time step, agents simultaneously inspect their neighborhoods and compute two utility functions, associated with products 0 and 1 , that represent a weighted average between their intrinsic preference and the choice of their neighbors,

$$
\begin{aligned}
& f_{n}^{0}(\boldsymbol{u}(t))=-\left(1-\lambda_{n}\right) \frac{q_{n}}{2}+\lambda_{n}\left[1-\frac{1}{\left|\square_{n}\right|} \sum_{n^{\prime} \in \square_{n}} u_{n^{\prime}}(t)\right], \\
& f_{n}^{1}(\boldsymbol{u}(t))=\left(1-\lambda_{n}\right) \frac{q_{n}}{2}+\frac{\lambda_{n}}{\left|\square_{n}\right|} \sum_{n^{\prime} \in \square_{n}} u_{n^{\prime}}(t) .
\end{aligned}
$$

Once the utility functions have been computed, each agent selects a product at time $t+1$ according to a Bernoulli distribution whose mean depends upon the difference between the utility functions at time $t$. More precisely, let

$$
\Delta f_{n}(\boldsymbol{u}(t))=f_{n}^{1}(\boldsymbol{u}(t))-f_{n}^{0}(\boldsymbol{u}(t))
$$

then the $n$th agent's choice is determined via the following conditional distribution:

$$
\begin{aligned}
& p\left(u_{n}(t+1)=1 \mid \boldsymbol{u}(t)=\boldsymbol{v}\right)=\frac{\exp \left[\beta \Delta f_{n}(\boldsymbol{v}(t))\right]}{\exp \left[-\beta \Delta f_{n}(\boldsymbol{v}(t))\right]+\exp \left[\beta \Delta f_{n}(\boldsymbol{v}(t))\right]}, \\
& p\left(u_{n}(t+1)=0 \mid \boldsymbol{u}(t)=\boldsymbol{v}\right)=\frac{\exp \left[-\beta \Delta f_{n}(\boldsymbol{v}(t))\right]}{\exp \left[-\beta \Delta f_{n}(\boldsymbol{v}(t))\right]+\exp \left[\beta \Delta f_{n}(\boldsymbol{v}(t))\right]}
\end{aligned}
$$

for $n=1, \ldots, N$ and $\beta \in \mathbb{R}$. The evolution of the system is best understood by inspecting the utility functions (2.3). The function $f_{n}^{1}$, for instance, is formed by two contributions: the first addend pertains to the perceived quality of product 1; the second addend accounts for the neighborhood's influence, since this term is proportional to the number of purchases of product 1 in the neighborhood. The relative importance of the two contributions is determined by the parameter $\lambda_{n}$.

The ABM described by (2.3)-(2.4), completed by initial conditions and explicit expressions for means and standard deviations in (2.1), defines an evolution equation that we will formally denote by

$$
\begin{aligned}
& \boldsymbol{u}(t+1)=\boldsymbol{\varphi}\left(\boldsymbol{u}(t) ; \gamma, \boldsymbol{\omega}^{n}\right), \quad t \in \mathbb{Z}_{+}, \quad \boldsymbol{u} \in \mathbb{B}^{N}, \quad \boldsymbol{\gamma} \in \mathbb{R}^{Q}, \\
& \boldsymbol{u}(0)=\boldsymbol{u}_{0},
\end{aligned}
$$

where we have collected in $\gamma$ the microscopic parameters

$$
\gamma=\left(q_{1}, \ldots, q_{n}, \lambda_{1}, \ldots, \lambda_{n}, \beta\right),
$$

and $\omega^{n}$ denotes the set of random choices that were made by the agents during this time step. 
Henceforth, we will refer to (2.5) as the lock-in model, implying that the agents behave as specified in (2.1)-(2.4). Unless otherwise stated, we shall assume all-to-all coupling, that is, $\square_{n}=\{1, \ldots, N\}$ for all $n$, and random Bernoulli-distributed initial conditions with average 0.5 :

$$
u_{0 n} \sim \mathcal{B}(u ; 0.5), \quad u_{0 n} \text { i.i.d. for } n=1, \ldots, N
$$

Remark 2.1 (interpretation of the coordinates $\boldsymbol{r}_{n}$ ). Since we have chosen all-to-all coupling and agents are identically coupled via their mean preference (see (2.3)), it would be misleading to interpret $\boldsymbol{r}_{n}=\left(x_{n}, y_{n}\right)$ as a location in physical space: with this type of coupling the spatial position of the agents does not play any role in the evolution of the system. However, from (2.1)-(2.2) we see that $x_{n}$ is used to order agents by their mean preference, via the sigmoidal function $\mu\left(x_{n}\right)$. Such ordering is of course arbitrary, but it allows us to make a distinction between two cases: homogeneous agents, when the distribution of the quality parameter $q_{n}$ is the same for all agents $(\Delta \mu=0)$, and heterogeneous agents, when the average perceived quality varies within the population. In the remainder of this paper, agents will be presented on a two-dimensional lattice only for visualization purposes, and the reader should interpret $x_{n}$ as a position in preference space - not physical space. Such preference space is parametrized by the single coordinate $x_{n}$, as $y_{n}$ does not play a role in our simulations.

Remark 2.2 (deterministic lock-in model). The evolution of the lock-in model is stochastic, since agents' choices are determined via (2.4). However, it is possible to study a deterministic evolution by considering the limit $\beta \rightarrow \infty$. In this case, agents purchase their product according to

$$
u_{n}(t+1)=\left\{\begin{array}{ll}
u_{n}(t) & \text { if } f_{n}^{0}(\boldsymbol{u}(t))=f_{n}^{1}(\boldsymbol{u}(t)), \\
0 & \text { if } f_{n}^{0}(\boldsymbol{u}(t))>f_{n}^{1}(\boldsymbol{u}(t)), \\
1 & \text { if } f_{n}^{0}(\boldsymbol{u}(t))<f_{n}^{1}(\boldsymbol{u}(t)),
\end{array} \quad n=1, \ldots, N\right.
$$

In passing we note that, in the limit $\beta \rightarrow \infty, \Delta f_{n} \rightarrow 0$, equations (2.4) give a random choice and assign equal probability to 0 and 1 ; to make the model deterministic, we then prescribe that if $f_{n}^{0}(\boldsymbol{u}(t))=f_{n}^{1}(\boldsymbol{u}(t))$, the agent sticks with its previous decision. Then, $u_{n}(t+1)$ is a deterministic function of $\boldsymbol{u}(t)$ and of the agent's parameters $\lambda_{n}, q_{n}$. Even in that case, $\boldsymbol{u}(t)$ remains a random variable, since $\lambda_{n}, q_{n}$ and the initial condition $\boldsymbol{u}_{0}$ are randomly distributed according to (2.1) and (2.7), respectively. Our model additionally differs from the original model of Garlick and Chli [24] in two ways. First, the model in [24] is a deterministic lock-in model with no heterogeneity in the agent's preferences, $\Delta \mu=0$. Second, we rescaled the utility function so as to include a single parameter $q_{n}$ for the perceived quality, as opposed to having separate parameters for products 1 and 0 .

Remark 2.3 (possible model extensions). Different types of coupling can be considered for the agents. In addition to the all-to-all coupling adopted in the present paper, nearest-neighbor [24] and static/dynamic small-world couplings [64] are also possible. Considering nearest-neighbor coupling or agent motility would effectively introduce a genuine spatial dependence into the system (see Remark 2.1). More realistic models can also be obtained if the agents adapt their parameters $q_{n}$ and $\lambda_{n}$ as time varies, so that they can change their opinion about the products or their attitude toward the neighborhood.

Copyright (c) by SIAM. Unauthorized reproduction of this article is prohibited. 
Table 1

Parameter values for the lock-in model simulations of Figures 1 and 4. Experiments are done with all-to-all coupling and random Bernoulli-distributed initial conditions (2.7).

\begin{tabular}{cccccccc}
\hline Experiment & $\bar{\mu}$ & $\Delta \mu$ & $\alpha$ & \multicolumn{1}{c}{$\bar{\xi}$} & \multicolumn{1}{c}{$\bar{\zeta}$} & \multicolumn{1}{c}{$\bar{\zeta}$} & $\beta$ \\
\hline E1 & 0 & 0 & 0 & 0.236 & 0.05 & 0.0167 & 10 \\
E2 & 0 & 0 & 0 & 0.236 & 0.5 & 0.167 & 10 \\
E3 & 0 & 1 & 5.0 & 0.236 & 0.5 & 0.167 & 10 \\
E4 & 0 & 1 & 0.5 & 0.236 & 0.5 & 0.167 & 10 \\
\hline
\end{tabular}

(a)

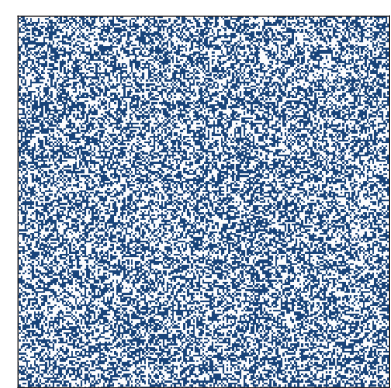

(c)

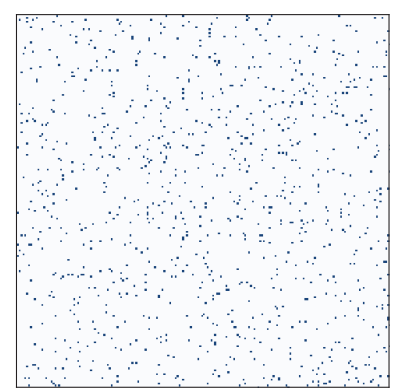

(b)

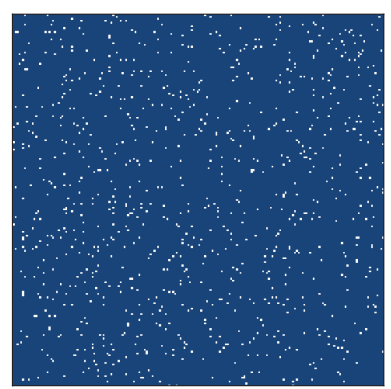

(d)

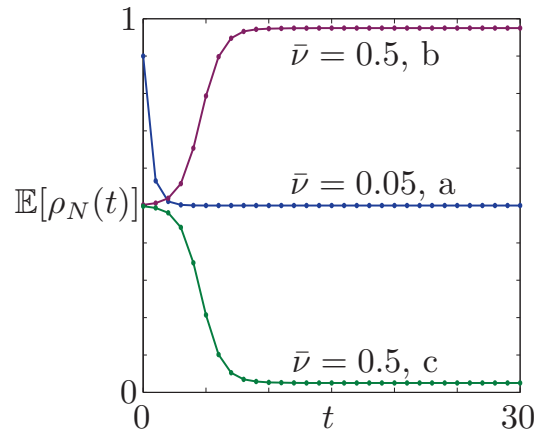

Figure 1. Homogeneous states of the lock-in model (2.1)-(2.4) with $N=200^{2}$ agents, corresponding to experiments $\mathrm{E} 1$ and $\mathrm{E} 2$ in Table 1. White and blue dots represent agents purchasing product 0 and 1 , respectively. The lock-in model is initialized sampling a Bernoulli distribution (2.7), and it is iterated for 30 time steps. (a) Mixed state obtained with experiment E1. (b) Global lock-in of product 1, obtained with one realization of E2. (c) Global lock-in of product 0, obtained with a second realization of E2. (d) Ensemble average of the average purchase $\rho_{N}=\sum_{n} u_{n} / N$ over 2000 realizations as a function of time for simulations of (a), (b), and (c); the experiment of (a) is here repeated with initial conditions $u_{0 n} \sim \mathcal{B}(u ; 0.9)$ for $n=0, \ldots, N$, showing that the mixed state is the unique macroscopic stable equilibrium for $\bar{\nu}=0.05$.

2.2. Simulation-based study of the lock-in system. We now discuss microscopic numerical simulations that motivate our choice of the macroscopic state variables. In the following numerical experiments, we iterate the lock-in model (2.5) with initial condition (2.7) for the choices of the parameter distributions (2.1) specified in Table 1. This leads naturally to

Copyright ( ) by SIAM. Unauthorized reproduction of this article is prohibited. 
the introduction of a set of macroscopic variables, which will be defined more precisely in section 2.3.

2.2.1. Globally locked-in states with homogeneous agents. With the first two experiments, using parameter sets E1 and E2, we find homogeneous macroscopic solutions corresponding to globally locked-in states. In Figures $1(\mathrm{a})-1(\mathrm{c})$ we show one mixed state obtained in E1 and two locked-in states obtained in E2. In each realization of these experiments, we obtain different final states since the evolution is stochastic and the initial condition $\boldsymbol{u}_{0}$ as well as the microscopic parameters $\gamma$ are randomly distributed. We recall that agents are presented on a two-dimensional lattice for visualization purposes, but their position $\boldsymbol{r}_{n}$ on the lattice does not influence the dynamics (see Remark 2.1).

In experiment E1, we set the agent's parameters so that the average perceived quality of products 0 and 1 is identical and the tendency to follow the neighborhood is low (see Table 1 and Figure 1(a)). The resulting state is a mixed state, with an even distribution of final products. In experiment E2, we increase the average and variance of the coupling (Figures 1(b)-1(c)) and observe two locked-in states (each equally likely to occur) in which almost all agents continually purchase one product, irrespective of its perceived quality. Indeed, since $\bar{\mu}=\Delta \mu=0$, we expect that on average only half of the agents have a preference for the dominant product, whereas agents in the remaining half purchase a product that they consider worse in terms of quality. As the experiment is repeated, we can get lock-in of either product, owing to the stochasticity of the evolution and the randomness of microscopic parameters and initial conditions. These results are in accordance with what was reported by Garlick and Chli [24] for a deterministic lock-in model with all-to-all coupling (see also Remark 2.2) and reinforce the similarity between the lock-in ABM and other Ising-type sociophysical models available in the literature [10].

It is natural to seek a characterization of the lock-in model in terms of a simple macroscopic variable and to interpret the statistical equilibria obtained as steady states of a suitably defined dynamical system. In Figure 1(d) we begin introducing such a characterization: we repeat 2000 times the numerical simulations that led to each of the states in panels (a), (b), (c), group each of the samples by their mean purchase

$$
\rho_{N}(t)=\frac{1}{N} \sum_{n} u_{n}(t) \in \mathbb{Q}_{N}, \quad t \in \mathbb{Z}_{+}, \quad \text { where } \mathbb{Q}_{N}=\left\{\frac{n}{N} \in \mathbb{Q} \mid 0 \leq n \leq N\right\},
$$

and plot the ensemble average of these means as a function of time. The macroscopic variable $\rho_{N}$ is a scalar, as the agent's preferences do not depend on $\boldsymbol{r}_{n}$. The plot in Figure 1(d) shows that, in this description, locked-in and mixed states are achieved rapidly, within just 10 iterations of the map. For a low value of the average coupling strength $\bar{\nu}$, the system reaches a single macroscopic state: from panel (d) we see that, in this region of parameter space, the mixed state is attracting even if the initial conditions are close to a fully locked-in state, that is, $u_{0 n} \sim \mathcal{B}(u ; 0.9)$ for $n=1, \ldots, N$. Upon increasing the coupling strength, we find two new macroscopic states, suggesting the presence of a pitchfork bifurcation at the macroscopic level.

However, a more careful inspection shows that these macroscopic locked-in solutions are not stable steady states but rather coarse metastable states: it is indeed known that, in

Copyright (c) by SIAM. Unauthorized reproduction of this article is prohibited. 
(a)

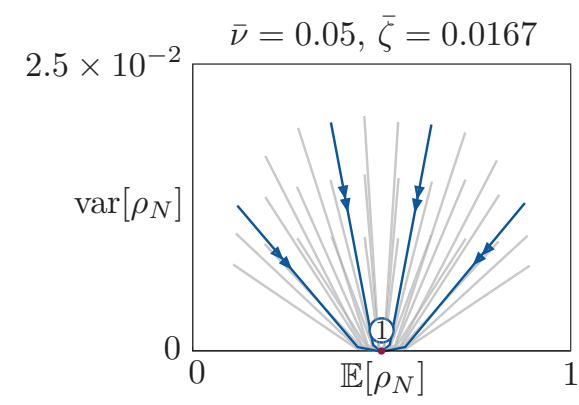

(c)

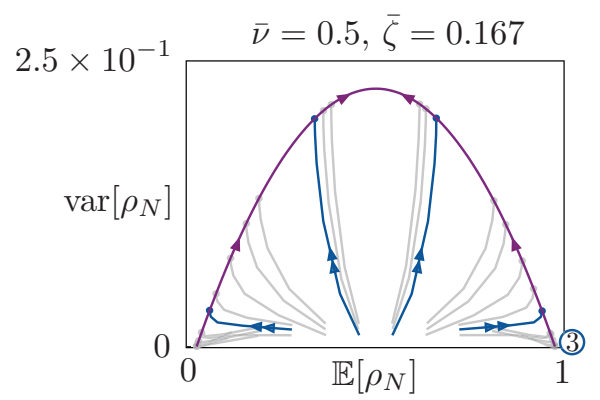

(b)

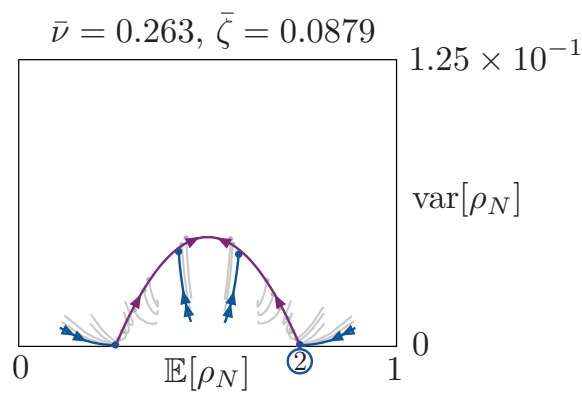

(d)
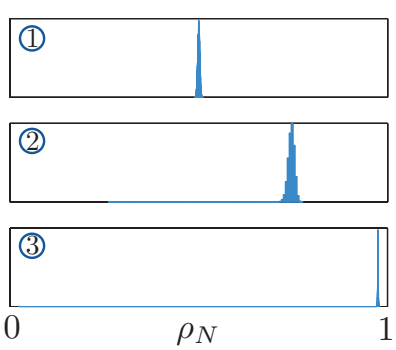

Figure 2. Expectation and variance of the average agents' choice $\rho_{N}(t)=\sum_{n} u_{n}(t)$ starting from different initial conditions. For each trajectory on the $\left(\mathbb{E}\left[\rho_{N}\right]\right.$, var $\left.\left[\rho_{N}\right]\right)$-plane, we initialize $M=10^{5}$ independent simulations for $N=200^{2}$ agents (as in Figure 1) with various initial conditions and iterate the lock-in model until $t=20$. (a) Low average coupling $\lambda_{n}$ (as in $\mathrm{E} 1$ of Table 1). (b) Intermediate value of the average coupling. (c) High value of the average coupling (as in E2). (d) Examples of final distributions of $\rho_{N}$.
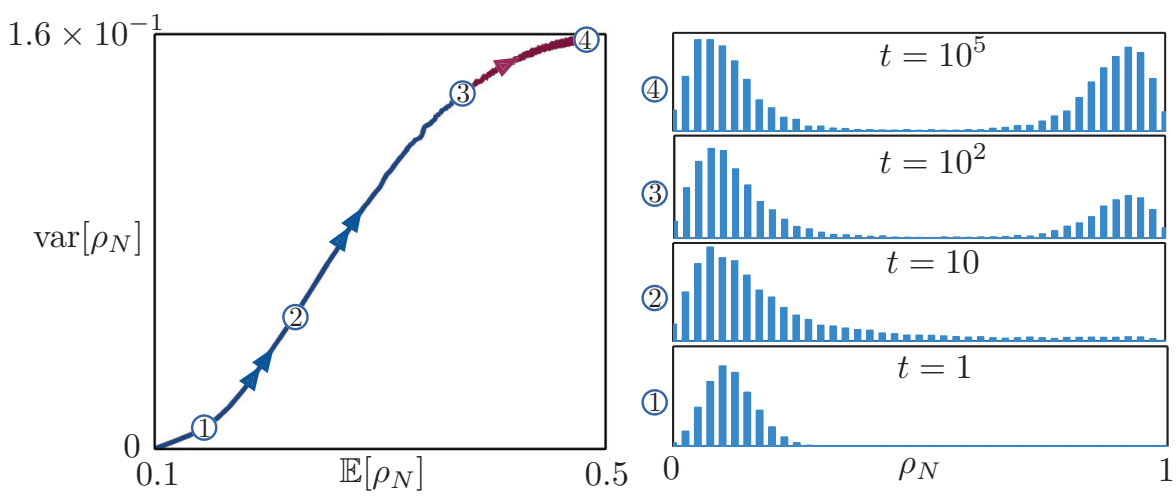

Figure 3. Expectation and variance of the average agents' choice $\rho_{N}(t)=\sum_{n} u_{n}(t) / N$ for the lock-in model with $N=40$ agents. See also the accompanying animation (96218_01.avi [local/web 2.06MB]). Left: We initialize $M=10^{4}$ realizations from $u_{0 n}=0.1$ for all $n$; the evolution on the $\left(\mathbb{E}\left[\rho_{N}\right]\right.$, var $\left.\left[\rho_{N}\right]\right)$-plane is plotted with a blue line for the first $10^{2}$ iterations and in magenta for the following iterations, until $t=10^{5}$. Right: Distributions of $\rho_{N}$ at various times. After a short transient, the evolution takes place on a slow manifold. Parameters: $\bar{\mu}=0, \Delta \mu=0, \alpha=0, \bar{\xi}=0.235, \bar{\nu}=0.3, \bar{\zeta}=0.03, \beta=8$.

Copyright ( $\odot$ by SIAM. Unauthorized reproduction of this article is prohibited. 
sociophysical models, the lifetime of metastable states is linked to finite system size [10]. In Figure 2, we repeat similar computations and monitor $\mathbb{E}\left[\rho_{N}\right]$ and $\operatorname{var}\left[\rho_{N}\right]$ as a function of time. This time we prepare realizations with a different initial expectation and variance, so as to plot several orbits on the $\left(\mathbb{E}\left[\rho_{N}\right], \operatorname{var}\left[\rho_{N}\right]\right)$-plane. A low average value of the coupling parameter $\lambda_{n}$ leads to a single steady state, as shown in Figure 2(a). For intermediate and high values of the coupling (Figures 2(b) and 2(c)), trajectories are quickly attracted to a slow manifold (purple curve) which, for these choices of parameters, is well approximated by a parabola. In equation-free terminology, the existence of a slow manifold in the $\left(\mathbb{E}\left[\rho_{N}\right], \operatorname{var}\left[\rho_{N}\right]\right)$-plane is referred to as slaving.

Asymptotic equilibria in Figure 2(a) have a small variance (they correspond to sharply peaked distributions with average equal to 0.5), while asymptotic equilibria in Figures 2(b) and 2(c) have a much higher variance. The latter distributions have means equal to 0.5 , but they are bimodal (as will be shown below).

In Figure 2, we iterate the lock-in model only until $t=20$, a time scale clearly suggested by the coarse solution curves of Figure 1(d). In fact, for these choices of the control parameters, the time scale of the drifting on the slow manifold is so long that it is not feasible to observe it with numerical computations; hence the magenta curves in Figures 2(b)-2(c) are obtained by fitting a parabola to the set of final points on the phase plane. In Figure 3 and the accompanying animation (96218_01.avi [local/web 2.06MB]), the system size and parameters have been adjusted to observe drifting on more affordable time scales $(N=40, \bar{\mu}=0, \Delta \mu=0$, $\alpha=0, \bar{\xi}=0.235, \bar{\nu}=0.3, \bar{\zeta}=0.03$, and $\beta=8$ ): the initial probability distribution of $\rho_{N}$ is a Dirac delta, which becomes a unimodal distribution with nonzero variance on time scales of order $t=10$ and drifts toward a bimodal distribution on time scales of order $t=10^{5}$. The system therefore always evolves toward a state with $\mathbb{E}\left[\rho_{N}\right]=1 / 2$. However, the difference between strong coupling and weak coupling is clearly visible: when the agents are weakly coupled, each individual realization of the system evolves to a mixed state with $\rho_{N}=1 / 2$, so there is no lock-in, whereas with strong coupling between the agents, each realization will display lock-in, and the initial condition determines which state the agents will be locked into. Due to microscopic stochasticity, the system only equilibrates over a very long time scale, over which a fraction of the realizations flips to the other locked-in state in the latter case.

As a consequence, even though these locked-in solutions are only metastable, it is still meaningful to characterize them as fixed points of an evolution map on intermediate timescales. Barkley, Kevrekidis, and Stuart studied metastable states in physical systems with similar properties and use the term moment map for the coarse evolution operator [5]. We shall return to this moment map for homogeneous steady states in section 3 , where we derive an approximate coarse evolution map for the lock-in model.

2.2.2. Fronts for heterogeneous agents. We now turn to heterogeneous states, which correspond to large-dimensional coarse maps and are more challenging to compute with equationfree methods. More specifically, we allow the average quality perception $\mu$ to vary within the population, as specified in (2.2): in experiments E3 and E4 we choose a nonzero $\Delta \mu$ and vary the steepness $\alpha$ of the sigmoidal function $\mu\left(x_{n}\right)$. In E3, for instance, we choose $\bar{\mu}=0$, $\Delta \mu=1$, and $\alpha=5$, so the agents are split into two factions of the same size: the coordinate $x$ parametrizes the agents' mean preferences, so agents with negative $x$ like product 0 and

Copyright (c) by SIAM. Unauthorized reproduction of this article is prohibited. 
(a)
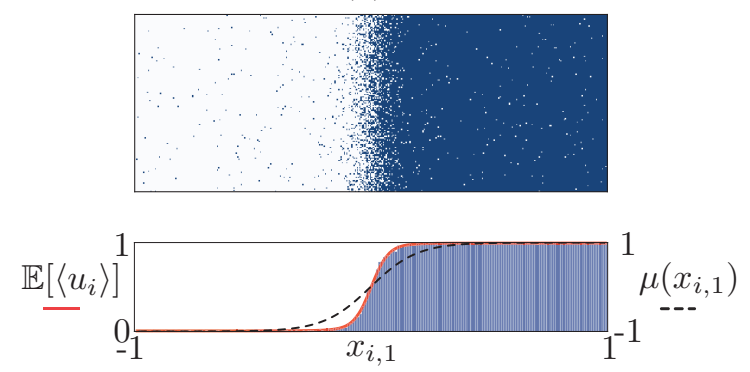

(b)

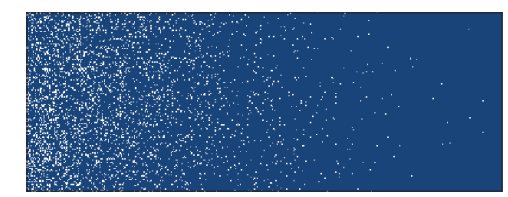

(c)
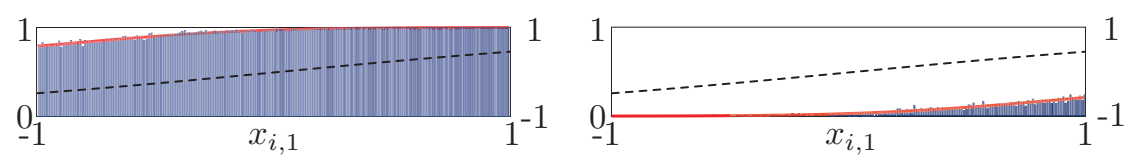

Figure 4. Heterogeneous states of the lock-in model (2.1)-(2.4) with $N=400 \times 150$ agents, corresponding to experiments E3 and E4 in Table 1. See also the accompanying animations (96218_02.avi [local/web 3.58MB] and 96218_03.avi [local/web 6.00MB]). The square $[-1,1]^{2}$ has been scaled to a rectangle for visualization purposes, and we use $x_{i, j}$ to indicate the position on the lattice. The lock-in model is initialized sampling a Bernoulli distribution (2.7), and it is iterated for 30 time steps. (a) In experiment E3 a stable interface is formed between two locked-in states (top); macroscopic states are obtained by averaging along the y axis (bottom, blue histogram) and then taking an ensemble average with respect to 2000 realizations (bottom, red curve, left axis). The resulting macroscopic state is a front connecting a macroscopic 0-state with a macroscopic 1-state; the front is sharper than the profile $\mu$ of the average quality perception parameters $q_{n}$ (bottom, dashed black curve, right axis). (b)-(c) In experiment $\mathrm{E} 4$, the slope of the profile $\mu$ is varied; the macroscopic front loses stability, giving rise to two stable heterogeneous states featuring pockets of resistance.

agents with positive $x$ favor product 1 . In E4, the sigmoid $\mu(x)$ is less steep, meaning that the two factions still have the same size, but there are fewer zealots.

For these experiments we choose a lattice of $400 \times 150$ agents, initialize the system with the Bernoulli distribution with success probability 0.5, and evolve the map for 30 iterations. As in the homogeneous case, the two-dimensional lattice is used only for visualization purposes, and the position on the lattice should not be interpreted as a physical location but as a position in preference space (see Remark 2.1).

Figure 4(a) shows the results of experiment E3, for which $\alpha=5$. As expected, the inhomogeneity in the distribution of the average quality perception parameters $q_{n}$ induces the formation of a pattern. In the accompanying animation (96218_02.avi [local/web 3.58MB]) we initialize the system using a slightly different initial condition, $u_{0 n}=H\left(x_{n}\right)$, where $H$ is the Heaviside function. The state represented in Figure 4(a) is the only attracting solution in this region of parameter space.

Copyright (c) by SIAM. Unauthorized reproduction of this article is prohibited. 
We shall abandon for a moment the lexicographical ordering used so far and denote the agents' purchases as $u_{i, j}$. To obtain a macroscopic description of the state in Figure 4(a), we compute averages of the purchases along the $y$-axis, $\left\langle u_{i}\right\rangle$, and then take an ensemble average with respect to 2000 realizations of the same experiment (red curve in panel (a)). The resulting macroscopic state is a front connecting a macroscopic 0-state to a 1-state: the front is parametrized by the agent's mean preference or, equivalently, by $x$. For convenience, we also plot $\mu\left(x_{i, 1}\right)$ and compare it to the macroscopic front, noting that the final macroscopic steady state is sharper than the profile of $\mu$. As we decrease $\alpha$, the macroscopic front persists and becomes flatter, until a critical point at which two new inhomogeneous states emerge (see the accompanying animation (96218_03.avi [local/web 6.00MB])). Such states, obtained with experiment E4 and shown in Figures 4(b) and 4(c), are related via the transformation

$$
\mathbb{E}\left[\left\langle u_{i}\right\rangle\right] \mapsto-\mathbb{E}\left[\left\langle u_{-i}\right\rangle\right]+1 .
$$

The scenario described above suggests that, as $\alpha$ is decreased, the front of Figure 4(a) undergoes a symmetry-breaking bifurcation at the macroscopic level. To the best of our knowledge, this type of transition has not been observed before in studies of opinion formation models. In the following sections, we will give a more precise definition of the macroscopic variables chosen to describe the lock-in systems for both homogeneous and inhomogeneous states and then proceed to perform a numerical bifurcation analysis of the corresponding states.

2.3. Macroscopic level description. Let us consider the lock-in model for fixed values of the microscopic parameters $\gamma \in \mathbb{R}^{Q}$, which are randomly distributed via (2.1) and remain constant at all times. ${ }^{1}$ Then, we denote by $\chi(\boldsymbol{u}, t \mid \gamma)$ the probability distribution of the vector $\boldsymbol{u}(t)$, given these microscopic parameters $\boldsymbol{\gamma}$. Now, considering that the microscopic parameters themselves are distributed according to a probability distribution $\psi(\boldsymbol{\gamma} ; \boldsymbol{\Gamma})$ that depends on a (small) number of macroscopic parameters $\boldsymbol{\Gamma}=(\bar{\mu}, \Delta \mu, \alpha, \bar{\xi}, \bar{\nu}, \bar{\zeta}) \in \mathbb{R}^{P}$, we can define the joint probability distribution of microscopic parameters and states as

$$
p(\boldsymbol{u}, \boldsymbol{\gamma}, t)=\chi(\boldsymbol{u}, t \mid \gamma) \psi(\boldsymbol{\gamma} ; \boldsymbol{\Gamma}) .
$$

We note that, to simplify notation, we often omit the explicit dependence of $\psi$ on $\boldsymbol{\Gamma}$. The probability distribution $P(\boldsymbol{u}, t)$ for an average agent at time $t$ is then obtained by integrating over all possible microscopic parameter values,

$$
P(\boldsymbol{u}, t)=\int_{\mathbb{R}^{Q}} p(\boldsymbol{u}, \boldsymbol{\gamma}, t) d \boldsymbol{\gamma} .
$$

We can formally write the time evolution of $P(\boldsymbol{u}, t)$ as

$$
P(\boldsymbol{u}, t+1)=\int_{\mathbb{R}^{Q}} \int_{\mathbb{B}^{N}} \Psi(\boldsymbol{u} \mid \boldsymbol{v}, \boldsymbol{\gamma}) p(\boldsymbol{v}, t, \boldsymbol{\gamma}) d \boldsymbol{v} d \boldsymbol{\gamma},
$$

where $\Psi(\boldsymbol{u} \mid \boldsymbol{v}, \gamma)$ represents the transition kernel, that is, the probability distribution of the state at time $t+1$ given that the system was in $\boldsymbol{v}$ at time $t$ with constant microscopic parameters $\gamma \cdot{ }^{2}$

\footnotetext{
${ }^{1}$ The vector $\gamma$, as given by (2.6), also contains the deterministic parameter $\beta$, which has been omitted here for simplicity.

${ }^{2}$ The transition kernel depends explicitly on time: $\Psi(\boldsymbol{u}, t+1 \mid \boldsymbol{v}, t, \boldsymbol{\gamma})$. However, time dependence has been omitted here to simplify the notation.
}

Copyright (c) by SIAM. Unauthorized reproduction of this article is prohibited. 
The macroscopic state $\boldsymbol{U}(t)=\left(U_{n}(t)\right)_{n=1}^{N}$ that was described informally in section 2.2 is the ensemble average of a large number of realizations, each with different microscopic parameters. In the limit of infinitely many realizations $(M \rightarrow \infty)$, this corresponds to taking the expectation of $\boldsymbol{u}$ with respect to the probability distribution $P(\boldsymbol{u}, t)$ of the microscopic realizations,

$$
\boldsymbol{U}(t):=\mathbb{E}[\boldsymbol{u}(t)]=\int_{\mathbb{B}^{N}} \boldsymbol{u} P(\boldsymbol{u}, t) d \boldsymbol{u}
$$

leading to the evolution map

$$
\boldsymbol{U}(t+1)=\int_{\mathbb{B}^{N}} \boldsymbol{u}\left[\int_{\mathbb{R}^{Q}} \int_{\mathbb{B}^{N}} \Psi(\boldsymbol{u} \mid \boldsymbol{v}, \boldsymbol{\gamma}) p(\boldsymbol{v}, \boldsymbol{\gamma}, t) d \boldsymbol{v} d \boldsymbol{\gamma}\right] d \boldsymbol{u}
$$

Clearly, the macroscopic evolution above cannot be written as a closed form equation that depends explicitly on $\boldsymbol{U}(t)$ unless one makes a closure approximation that specifies $p(\boldsymbol{u}, \boldsymbol{\gamma}, t)$ as a function of $\boldsymbol{U}(t)$. The focus of the present paper is to obtain bifurcation diagrams for fixed points of the coarse map (2.10). The algorithm that will be presented in section 4 is a procedure to impose the aforementioned closure approximation numerically.

Remark 2.4 (low-dimensional coarse descriptions). Our macroscopic description is highdimensional in that $\boldsymbol{U}$ is a vector with $N$ entries. Lower-dimensional descriptions can be obtained expressing $\boldsymbol{U}$ in terms of a coarse polynomial or spectral basis [26, 51, 41]. Since we aim to develop a numerical framework suitable for high-dimensional coarse systems (and applicable to the low-dimensional descriptions as well), we will continue to use simple agentwise coarse variables in this paper.

Remark 2.5 (discrete distributions). Since $\boldsymbol{u} \in \mathbb{B}^{N}$, the microscopic state belongs to a discrete set of possible admissible states with cardinality $2^{N}$. Thus, the probability distribution can be written as

$$
P(\boldsymbol{u}, t)=\sum_{\boldsymbol{v} \in \mathbb{B}^{N}} P^{*}(\boldsymbol{v}, t) \delta(\boldsymbol{u}-\boldsymbol{v}),
$$

and integrals of the type (2.9) should be interpreted as a discrete sum,

$$
\int_{\mathbb{B}^{n}} f(\boldsymbol{u}) P(\boldsymbol{u}, t) d \boldsymbol{u}=\sum_{\boldsymbol{u} \in \mathbb{B}^{n}} f(\boldsymbol{u}) P^{*}(\boldsymbol{u}, t) .
$$

In other words, the integral is computed assigning to each possible configuration $\boldsymbol{u}$ a weight corresponding to its probability $P^{*}(\boldsymbol{v}, t)$. In practical computations, however, we will not be able to simulate all possible realizations $\boldsymbol{u}$, so we will approximate the integrals by a Monte Carlo estimate using $M \ll 2^{N}$ realizations,

$$
\int_{\mathbb{B}^{n}} f(\boldsymbol{u}) P(\boldsymbol{u}, t) d \boldsymbol{u} \approx \frac{1}{M} \sum_{m=1}^{M} f\left(\boldsymbol{u}^{m}\right),
$$

where $\boldsymbol{u}^{m}$ are sampled from the probability distribution $P(\boldsymbol{u}, t)$.

In section 2.2 we have introduced homogeneous and inhomogeneous macroscopic states that we are now ready to characterize by means of coarse bifurcation analysis: for the former, a simple one-dimensional coarse description exists and will be discussed in the following section; for the latter, we will use equation-free bifurcation analysis, which will be the subject of sections $4-6$.

Copyright ( $\odot$ by SIAM. Unauthorized reproduction of this article is prohibited. 
3. Homogeneous macroscopic states. We begin by characterizing homogeneous macroscopic states, which are described in terms of the average purchase

$$
\rho_{N}(t)=\frac{1}{N} \sum_{n} u_{n}(t) \in \mathbb{Q}_{N}
$$

For each $t \in \mathbb{Z}^{+}, \rho_{N}(t)$ is a random variable, whose probability distribution is denoted by

$$
\bar{P}\left(\rho_{N}, t\right)=\int_{\Sigma_{\rho_{n}}} P(\boldsymbol{u}, t) d \boldsymbol{u}, \quad \Sigma_{\rho_{n}}=\left\{\boldsymbol{u} \in \mathbb{B}^{N} \mid \rho_{N}=\frac{1}{N} \sum_{n} u_{n}\right\} .
$$

The numerical simulations of Figure 2 lead us to search for a coarse evolution map whose fixed points correspond to the homogeneous metastable locked-in states of the lock-in model. Following [5], we search for a first moment map, that is, a map that closes at the level of the first moment $\mathbb{E}\left[\rho_{N}(t)\right]$ of the probability distribution $\bar{P}\left(\rho_{N}, t\right)$. In this section we show that a first moment map can be found explicitly under suitable hypotheses.

Lemma 3.1. Let us consider the lock-in model (2.5) under the following hypotheses:

1. All-to-all coupling, $\square_{n}=\{1, \ldots, N\}$ for all $n$.

2. Deterministic evolution, that is, $\beta \rightarrow \infty$.

3. Deterministic tendency to follow the neighbors

$$
\lambda_{n} \sim \delta(\lambda-\bar{\nu}), \quad \bar{\nu} \in(0,1), \quad n=1, \ldots, N .
$$

4. Homogeneous distribution of the quality perception $q_{n} \sim \mathcal{N}(q ; \bar{\mu}, \bar{\xi}), q_{n}$ i.i.d.

Further, let $\rho_{N}(t)$ be the mean purchase as defined in (3.1). Then, in the limit as $N \rightarrow \infty$, we have

$$
\mathbb{E}\left[\rho_{\infty}(t+1)\right]=\frac{1}{2} \operatorname{erfc}\left[\frac{1}{\bar{\xi} \sqrt{2}}\left(\bar{\nu} \frac{1-2 \mathbb{E}\left[\rho_{\infty}(t)\right]}{1-\bar{\nu}}-\bar{\mu}\right)\right]+\mathcal{O}\left(\operatorname{var}\left[\rho_{\infty}(t)\right]\right)
$$

Proof. Hypotheses 1 and 2 imply that the state of an individual agent $u_{n}(t+1)$ is a deterministic function of $\rho_{N}(t)$ and the individual perceived quality $q_{n}$, which are both random quantities (see Remark 2.2). Furthermore, hypothesis 3 implies $^{3}$

$$
u_{n}\left(t+1 \mid \rho_{N}(t), q_{n}, \bar{\nu}\right)=\left\{\begin{array}{ll}
1 & \text { if } q_{n}>\frac{\bar{\nu}}{1-\bar{\nu}}\left(1-2 \rho_{N}(t)\right), \\
0 & \text { otherwise }
\end{array} \quad n=1, \ldots, N\right.
$$

Next, let us denote by $\bar{p}_{n}\left(\rho_{N}, t, q\right)$ the joint probability of obtaining a mean purchase $\rho_{N}$ and a perceived quality $q$ for agent $n$. Owing to hypotheses 1 and 4 , the agentwise expectation of $u_{n}$ with respect to all possible realizations of the microscopic parameters,

$$
\mathbb{E}\left[u_{n}(t+1)\right]=\int_{\mathbb{Q}_{N}} \int_{\mathbb{R}} u_{n}\left(t+1 \mid \rho_{N}, q, \bar{\nu}\right) \bar{p}_{n}\left(\rho_{N}, t, q\right) d q d \rho_{N},
$$

\footnotetext{
${ }^{3}$ We use (2.8) and omit the case $f_{n}^{0}(\boldsymbol{u}(t))=f_{n}^{1}(\boldsymbol{u}(t))$, which corresponds to an event of measure 0 .
} 
is the same for all $n$, since $\bar{p}_{n}\left(\rho_{N}, t, q\right)=\bar{p}\left(\rho_{N}, t, q\right)$, independently of $n$. Hence,

$$
\mathbb{E}\left[\rho_{N}(t)\right]=\frac{1}{N} \sum_{n} \mathbb{E}\left[u_{n}(t)\right]=\mathbb{E}\left[u_{n}(t)\right] .
$$

Similarly, we write $\bar{p}\left(\rho_{N}, t, q\right)=\tilde{p}\left(\rho_{N}, t \mid q\right) \mathcal{N}(q ; \bar{\mu}, \bar{\xi})$, in which $\mathcal{N}(q ; \bar{\mu}, \bar{\xi})$ is the probability density of the Gaussian distribution from which $q$ was drawn. In the limit as $N$ tends to infinity, we moreover have that $\tilde{p}\left(\rho_{N}, t \mid q\right)=\bar{P}\left(\rho_{N}, t\right)$, as the mean purchase is then independent of a specific agent's perceived quality. We then use (3.2) to obtain

$$
\begin{aligned}
\mathbb{E}\left[\rho_{N}(t+1)\right] & =\mathbb{E}\left[u_{n}(t+1)\right] \\
& \approx \int_{\mathbb{Q}_{N}} \int_{\mathbb{R}} u_{n}\left(t+1 \mid \rho_{N}, q, \bar{\nu}\right) \bar{P}\left(\rho_{N}, t\right) \mathcal{N}(q ; \bar{\mu}, \bar{\xi}) d q d \rho_{N} \\
& =\int_{\mathbb{Q}_{N}}\left[\int_{\frac{\bar{\nu}\left(1-2 \rho_{N}(t)\right)}{1-\bar{\nu}}}^{\infty} \mathcal{N}(q ; \bar{\mu}, \bar{\xi}) d q\right] \bar{P}\left(\rho_{N}, t\right) d \rho_{N} \\
& =\int_{\mathbb{Q}_{N}} \frac{1}{2} \operatorname{erfc}\left[\frac{1}{\overline{\bar{\xi}} \sqrt{2}}\left(\bar{\nu} \frac{1-2 \rho_{N}(t)}{1-\bar{\nu}}-\bar{\mu}\right)\right] \bar{P}\left(\rho_{N}, t\right) d \rho_{N} \\
& =\mathbb{E}\left[\frac{1}{2} \operatorname{erfc}\left[\frac{1}{\bar{\xi} \sqrt{2}}\left(\bar{\nu} \frac{1-2 \rho_{N}(t)}{1-\bar{\nu}}-\bar{\mu}\right)\right]\right] \\
& :=\mathbb{E}\left[\Psi\left(\rho_{N}(t) ; \bar{\nu}, \bar{\mu}, \bar{\xi}\right)\right],
\end{aligned}
$$

where the expectation is taken over all possible values of $\rho_{N}$.

The equation above does not close at the level of $\mathbb{E}\left[\rho_{N}\right]$, since $\Psi$ is a nonlinear function of $\rho_{N}$, and therefore $\mathbb{E}\left[\Psi\left(\rho_{N}\right)\right] \neq \Psi\left(\mathbb{E}\left[\rho_{N}\right]\right)$. However, in the limit as $N \rightarrow \infty$, we can perform a Taylor expansion of $\psi(\rho)$ around $\mathbb{E}[\rho]$ (see $[32,66]$ ),

$$
\begin{aligned}
\mathbb{E}\left[\Psi\left(\rho_{\infty}\right)\right] & \approx \mathbb{E}\left[\Psi\left(\mathbb{E}\left[\rho_{\infty}\right]\right)+\Psi^{\prime}\left(\mathbb{E}\left[\rho_{\infty}\right]\right)\left(\rho_{\infty}-\mathbb{E}\left[\rho_{\infty}\right]\right)+\frac{1}{2} \Psi^{\prime \prime}\left(\mathbb{E}\left[\rho_{\infty}\right]\right)\left(\rho_{\infty}-\mathbb{E}\left[\rho_{\infty}\right]\right)^{2}\right] \\
& =\mathbb{E}\left[\Psi\left(\mathbb{E}\left[\rho_{\infty}\right]\right)\right]+\frac{1}{2} \Psi^{\prime \prime}\left(\mathbb{E}\left[\rho_{\infty}\right]\right) \mathbb{E}\left[\left(\rho_{\infty}-\mathbb{E}\left[\rho_{\infty}\right]\right)^{2}\right] \\
& =\Psi\left(\mathbb{E}\left[\rho_{\infty}\right]\right)+\mathcal{O}\left(\operatorname{var}\left[\rho_{\infty}\right]\right)
\end{aligned}
$$

which combined with (3.4) proves the assertion.

Remark 3.2. In Lemma 3.1 we assume that the deterministic coupling constant $\bar{\nu}$ is strictly between 0 and 1 , in order to exclude trivial dynamics. If $\bar{\nu}=0$, then (3.4) gives

$$
\mathbb{E}\left[\rho_{N}(t+1)\right]=\frac{1}{2} \operatorname{erfc}\left(-\frac{\bar{\mu}}{\bar{\xi} \sqrt{2}}\right), \quad t \in \mathbb{Z}_{+},
$$

that is, a microscopic equilibrium is reached after one time step and the corresponding macroscopic equilibrium does not depend upon initial conditions. This is to be expected, since $\bar{\nu}=0$ means that agents disregard information about their neighbors; therefore, initial conditions are not relevant to their choice.

On the other hand, if $\bar{\nu}=1$, we cannot directly apply (3.4). However, we have

$$
u_{n}(1)=\left\{\begin{array}{ll}
1 & \text { if } \sum_{n} u_{n}(0)>N / 2, \\
0 & \text { otherwise, }
\end{array} \quad n=1, \ldots, N,\right.
$$

Copyright (C) by SIAM. Unauthorized reproduction of this article is prohibited. 
and so the system achieves a microscopic locked-in equilibrium after one time step. If, as was done in the numerical experiments of Figure 1, the microscopic initial conditions are independent identically distributed (i.i.d.) variables, $u_{n}(0) \sim \mathcal{B}(u, 0.5)$ for all $n=1, \ldots, N$, we have $\sum_{n=1}^{N} u_{n}(0) \sim \operatorname{Binomial}(N, 0.5)$, and therefore

$$
\mathbb{E}[\rho(1)]=1-\operatorname{Pr}\left[\sum_{n=1}^{N} u_{n}(0) \leq N / 2\right]=1-\frac{1}{2^{N}} \sum_{i=0}^{\lfloor N / 2\rfloor}\left(\begin{array}{c}
N \\
i
\end{array}\right) .
$$

Lemma 3.1 suggests a simple way to derive a coarse evolution map: if the hypotheses of the lemma hold true and we are in the limit of infinitely many agents, we can choose $U=\mathbb{E}\left[\rho_{\infty}\right]=\mathbb{E}\left[u_{n}\right]$ as our coarse variable; then, to leading order, we obtain

$$
U(t+1)=\frac{1}{2} \operatorname{erfc}\left[\frac{1}{\bar{\xi} \sqrt{2}}\left(\bar{\nu} \frac{1-2 U(t)}{1-\bar{\nu}}-\bar{\mu}\right)\right]:=\Phi_{\mathrm{a}}(U(t) ; \bar{\nu}, \bar{\mu}, \bar{\xi}) .
$$

It is clear that this is only an approximate evolution map, as we have tacitly assumed that the probability distribution for $\rho_{N}(t)$ is unimodal and sharply peaked, so that $\rho_{\infty} \approx \mathbb{E}\left[\rho_{\infty}\right]$ and $\operatorname{var}\left[\rho_{\infty}\right] \approx 0$. The numerical simulations of section 2.2 (in particular Figure 2 ) show that this is a valid approximation on sufficiently short time scales. By analogy with [5], we expect that fixed points of this first moment map will inform us about metastable homogeneous states of the full lock-in model; hence we proceed to discuss fixed points of the map and their stability.

For simplicity, let us consider the case of equally perceived products, such that $\bar{\mu}=0$, and fixed standard deviation $\bar{\xi}$ and study fixed points $U_{*}$ of $\Phi_{\mathrm{a}}$ as $\bar{\nu}$ is varied. For all $\bar{\nu}$, the map possesses a fixed point at $U_{*}=1 / 2$, the mixed state, which is stable for $\bar{\nu}<\bar{\nu}_{\mathrm{c}}$, where $\bar{\nu}_{\mathrm{c}}$ is computed as

$$
\Phi_{\mathrm{a}}^{\prime}\left(1 / 2 ; \bar{\nu}_{\mathrm{c}}, 0, \bar{\xi}\right)=1 \quad \Rightarrow \quad \bar{\nu}_{c}=\frac{1}{1+\bar{\xi}^{-1} \sqrt{2 / \pi}}
$$

At the critical point, two new fixed points arise (corresponding to equilibria with increasingly high proportions of one product over the other), while the mixed state becomes unstable at a pitchfork bifurcation. Since we have an analytic expression for $\Phi_{\mathrm{a}}$, we can readily apply numerical continuation techniques and obtain the bifurcation diagram shown in Figure 5.

Similar considerations are valid if we assume that the population has a bias toward one of the products $(\bar{\mu} \neq 0)$. Then, the pitchfork breaks into two branches: one of them, corresponding to the product with higher average perceived quality, is stable for all values of the coupling $\bar{\nu}$, whereas the other one destabilizes at a saddle-node bifurcation. As expected, the basin of attraction of the locked-in state is larger for the product with a higher perceived quality.

To further demonstrate the relation between fixed points of $\Phi_{\mathrm{a}}$ and metastable states of the lock-in model, we refer the reader to Figures 1 and 3. In the bistable region of parameter space, each realization of the stochastic process evolves to a state that corresponds to one of the stable fixed points of the coarse evolution map over reasonably short time scales; these equilibria in Figure 1 correspond to the fixed points of the coarse evolution map in Figure 5. For a microscopic stochastic simulation starting close to such an equilibrium, this implies that there is a distribution of mean purchases, unimodal and sharply peaked around this population

Copyright (c) by SIAM. Unauthorized reproduction of this article is prohibited. 


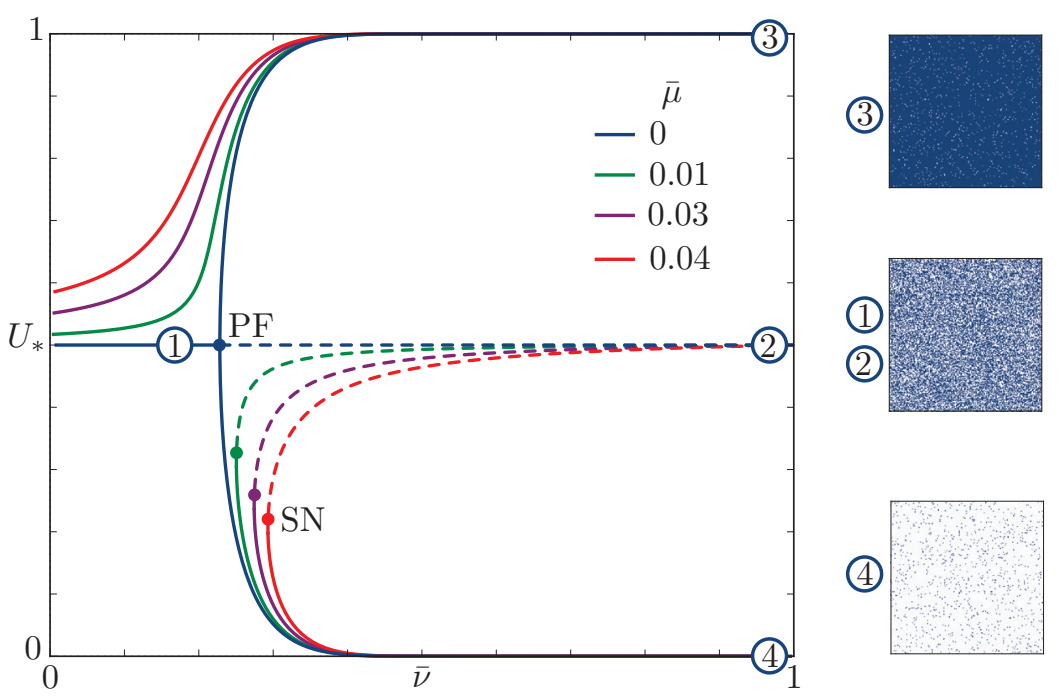

Figure 5. Fixed points of the approximate coarse map $\Phi_{a}$ as a function of the coupling parameter $\bar{\nu}$ for $\bar{\xi}=$ 0.236 and various values of $\bar{\mu}$. Solid (dashed) lines represent stable (unstable) coarse equilibria. Representative microscopic solutions are plotted on the right. If agents are unbiased on average $(\bar{\mu}=0)$, the mixed state becomes unstable at a pitchfork bifurcation (PF), attained at the critical value given by (3.7), and two locked-in states emerge. If agents have an average bias toward product $1(\bar{\mu}>0)$, the pitchfork breaks down, giving rise to a saddle node bifurcation (SN). A similar scenario occurs if $\bar{\mu}<0$ (not shown).

average, as can be observed in Figure 3. The equilibria computed from the analytic coarse evolution map approximate this distribution using a Dirac-delta distribution. However, over long time scales, both metastable states are equally likely to occur, as Figure 3 shows.

4. Equation-free Newton-Krylov method. In this section, we aim to obtain a numerical closure relation for the evolution of the $x$-dependent macroscopic state $\boldsymbol{U}=\left(U_{n}\right)_{n=1}^{N}$. In this case, an analytical closure approximation is no longer valid. We thus propose an equation-free method. We first outline the general principle of the equation-free methodology (section 4.1). Next, we describe the concrete lifting and restriction operators that will be used (section 4.2). The main algorithmic contribution of the present paper is the introduction of a weighted lifting and restriction operator that allows the accurate computation of Jacobian-vector products, as will be discussed in section 4.3.

4.1. Principle. As was shown in the previous sections, the lock-in model (2.5) consists, at the microscopic level, of individual agents whose state keeps evolving, owing to the probabilistic nature of their choices. Nevertheless, at the macroscopic level, the ensemble average (2.9) is seen to evolve to a metastable equilibrium. In this paper, we are interested in performing a bifurcation analysis at the macroscopic level, at which an exact, closed model is not available. The equation-free framework was developed for such tasks [37, 38].

The main building block in an equation-free method is the coarse time-stepper, which allows the performance of time-steps at the macroscopic level (defined by (2.10)), using only the simulation of $M$ realizations of the microscopic model (2.5). To achieve this, the procedure relies on the definition of two operators (lifting and restriction) that relate the microscopic 
and macroscopic levels of description. The lifting operator maps a macroscopic state to a microscopic one; that is, starting from a macroscopic state $\boldsymbol{U}$ and macroscopic parameters $\boldsymbol{\Gamma}$, it generates an ensemble,

$$
\boldsymbol{B}=\left[\boldsymbol{u}^{m}\right]_{m=1}^{M} \in \mathbb{B}^{N \times M},
$$

of $M$ realizations $\boldsymbol{u}^{m}(m=1, \ldots, M)$ of the microscopic state (each consisting of $N$ individual agents) from the ensemble average $\boldsymbol{U} \in \mathbb{R}^{N}$, as well as a set of $Q$ microscopic parameters for each agent, $\boldsymbol{A}=\left[\gamma^{m}\right]_{m=1}^{M}$, with $\gamma^{m} \in \mathbb{R}^{Q \times N}$, sampled from the parameter distributions (2.1) specified by the macroscopic parameters $\boldsymbol{\Gamma}=(\bar{\mu}, \Delta \mu, \alpha, \bar{\xi}, \bar{\nu}, \bar{\zeta}) \in \mathbb{R}^{P}$. We remark that, once sampled, the agents' parameters are kept fixed throughout the evolution step.

When generating random realizations for the microscopic state and parameters, applying the same lifting operator multiple times will give different results, depending on the precise random numbers that were generated during the process. We will denote this set of random numbers by $\boldsymbol{\omega} \in \boldsymbol{\Omega}$, in which the sample space $\boldsymbol{\Omega}$ represents all possible sets of random numbers that can be generated; it may be convenient to think of $\boldsymbol{\omega}$ as the set of seeds of all the random number generators involved. This leads to an operator of the form

$$
\begin{aligned}
\mathcal{L}: \mathbb{R}^{N} \times \mathbb{R}^{P} \times \boldsymbol{\Omega} & \longrightarrow \mathbb{B}^{N \times M} \times \mathbb{R}^{Q \times N \times M}, \\
(\boldsymbol{U}, \boldsymbol{\Gamma}, \boldsymbol{\omega}) & \longmapsto(\boldsymbol{B}, \boldsymbol{A}) .
\end{aligned}
$$

In what follows, we will also denote the lifting by

$$
(\boldsymbol{B}, \boldsymbol{A})=\left(\mathcal{L}_{u}(\boldsymbol{\omega}) \boldsymbol{U}, \mathcal{L}_{\gamma}(\boldsymbol{\omega}) \boldsymbol{\Gamma}\right)=\mathcal{L}(\boldsymbol{\omega})(\boldsymbol{U}, \boldsymbol{\Gamma}),
$$

to emphasize that $\boldsymbol{\omega}$ appears only as a parameter.

Remark 4.1 (dependence on the random event $\omega$ ). The explicit introduction of the parameter $\boldsymbol{\omega}$ may seem elaborate at first. Nevertheless, in the remainder of this paper, especially when discussing the computation of variance-reduced Jacobian-vector products in section 4.3, this notation will prove to be indispensable.

Remark 4.2 (closure approximation). The microscopic realizations have to be sampled from a probability distribution $P(\boldsymbol{u}, t)$ that is consistent with $\boldsymbol{U}(t)$; that is, we require $\int_{\mathbb{B}^{N}} \boldsymbol{u} P(\boldsymbol{u}, t) d \boldsymbol{u}=$ $\boldsymbol{U}(t)$. At this point, we have not yet specified what probability distribution $P(\boldsymbol{u}, t)$ will be used to this end. Choosing $P(\boldsymbol{u}, t)$ amounts to enforcing a closure approximation. In section 4.2, we will construct several lifting operators that perform this closure approximation numerically.

Conversely, the restriction operator maps a microscopic state to a macroscopic one; that is, it computes an appropriate ensemble average $\boldsymbol{U} \in \mathbb{R}^{N}$ of the $M$ realizations $\boldsymbol{B}=\left[\boldsymbol{u}^{m}\right]_{m=1}^{M} \in$ $\mathbb{B}^{N \times M}$ :

$$
\mathcal{R}: \mathbb{B}^{N \times M} \longrightarrow \mathbb{R}^{N}, \quad \boldsymbol{B} \longmapsto \boldsymbol{U}
$$

As a general principle, one expects the macroscopic state to be unchanged when performing lifting followed by restriction, that is,

$$
\mathcal{R} \circ \mathcal{L}_{u} \equiv \mathrm{Id} .
$$

Copyright (c) by SIAM. Unauthorized reproduction of this article is prohibited. 
In general, however, $\mathcal{L}_{u} \circ \mathcal{R} \neq \mathrm{Id}$, since it is impossible to recover exactly the microscopic information during lifting that was discarded during restriction. For the problem considered here, even ensuring (4.5) is nontrivial, because one cannot represent every possible value of $\boldsymbol{U}$ exactly as the ensemble average of $M$ microscopic realizations. Specific operators that circumvent this problem are proposed in section 4.2.

Once lifting and restriction operators have been constructed, a coarse time-stepper $\Phi_{T}^{M}$ to evolve the macroscopic state $\boldsymbol{U}$ over a time interval of length $T$ is constructed as a three-step procedure (lift-evolve-restrict), in which the microscopic evolution is simulated independently for each of the $M$ realizations; i.e.,

$$
\boldsymbol{U}(t+T)=\Phi_{T}^{M}(\boldsymbol{\omega})(\boldsymbol{U}(t) ; \boldsymbol{\Gamma})=\left(\mathcal{R} \circ \mathcal{E}_{T} \circ \mathcal{L}(\boldsymbol{\omega})\right)(\boldsymbol{U}(t) ; \boldsymbol{\Gamma}),
$$

with $\mathcal{L}(\boldsymbol{\omega})$ and $\mathcal{R}$ defined in (4.3) and (4.4), and $\mathcal{E}_{T}$ defined as

$$
\mathcal{E}_{T}: \mathbb{B}^{N \times M} \times \mathbb{R}^{Q \times N \times M} \longrightarrow \mathbb{B}^{N \times M},(\boldsymbol{B}, \boldsymbol{A}) \longmapsto\left[\varphi_{T}\left(\boldsymbol{u}^{m} ; \boldsymbol{\gamma}^{m}\right)\right]_{m=1}^{M},
$$

where we have denoted by $\varphi_{T}$ the $T$ th iterate of the lock-in map (2.5). Note that, in the limit $M \rightarrow \infty$, the coarse time-stepper approaches

$$
\boldsymbol{U}(t+T)=\int_{\mathbb{B}^{N}} \boldsymbol{u}\left[\int_{\mathbb{R}^{Q}} \int_{\mathbb{B}^{N}} \Psi_{T}(\boldsymbol{u} \mid \boldsymbol{v}, \boldsymbol{\gamma}) p(\boldsymbol{v}, t, \boldsymbol{\gamma} \mid \boldsymbol{U}(t), \boldsymbol{\Gamma}) d \boldsymbol{v} d \boldsymbol{\gamma}\right] d \boldsymbol{u}
$$

in which we have introduced the transition kernel $\Psi_{T}$ over a time interval $T$ and the probability distribution $p(\boldsymbol{v}, t, \gamma \mid \boldsymbol{U}(t), \boldsymbol{\Gamma})$ conditioned upon $(\boldsymbol{U}(t), \boldsymbol{\Gamma})$, from which the samples are taken. The interpretation of the coarse time-stepper as a numerical closure follows by comparing (4.8) with (2.10) and noticing that the right-hand side is completely determined by $\boldsymbol{U}(t)$ and $\boldsymbol{\Gamma}$, since the probability distribution $p(\boldsymbol{v}, t, \boldsymbol{\gamma} \mid \boldsymbol{U}(t), \boldsymbol{\Gamma})$ is conditioned upon $\boldsymbol{U}(t)$ and $\boldsymbol{\Gamma}$.

If the system (2.5) possesses macroscopic steady states, these can be found (for fixed macroscopic parameters $\boldsymbol{\Gamma}=\boldsymbol{\Gamma}_{*}$ ) by solving the nonlinear system

$$
\boldsymbol{F}\left(\boldsymbol{U}_{*}\right)=\boldsymbol{U}_{*}-\Phi_{T}^{M}(\boldsymbol{\omega})\left(\boldsymbol{U}_{*}, \boldsymbol{\Gamma}_{*}\right)=0
$$

for an appropriate choice of $M$ and $T$. This procedure allows the computation of unstable steady states that would not be reached by direct simulation. By adding a pseudoarclength condition, one can also perform continuation to obtain a branch of steady states as a function of a free parameter.

In each Newton iteration, one needs to solve a linear system involving the Jacobian of $\Phi_{T}^{M}$, denoted as $D \Phi_{T}^{M}(\boldsymbol{U} ; \boldsymbol{\Gamma})$. Since we do not have an explicit formula for $D \Phi_{T}^{M}(\boldsymbol{U} ; \boldsymbol{\Gamma})$, we are forced to use an iterative method (such as GMRES) that requires only Jacobian-vector products and to estimate such Jacobian-vector products using a finite difference approximation. However, we recall that, for a finite number of realizations $M$, the coarse time-stepper $\Phi_{T}^{M}$ is stochastic. Hence, repeating the same coarse time step with two sets of random numbers $\boldsymbol{\omega}_{1,2}$ gives different results. A standard Monte Carlo argument [8] reveals that

$$
\operatorname{var}\left[\Phi_{T}^{M}\left(\boldsymbol{\omega}_{1}\right)(\boldsymbol{U} ; \boldsymbol{\Gamma})-\Phi_{T}^{M}\left(\boldsymbol{\omega}_{2}\right)(\boldsymbol{U} ; \boldsymbol{\Gamma})\right] \leq C \frac{1}{M},
$$

Copyright $\odot$ by SIAM. Unauthorized reproduction of this article is prohibited. 
resulting in typical deviations of $\mathcal{O}(1 / \sqrt{M})$. Then, estimating Jacobian-vector products using the simple finite difference formula

$$
\begin{aligned}
D \Phi_{T}^{M}(\boldsymbol{U} ; \boldsymbol{\Gamma}) \boldsymbol{V} & \approx \frac{\Phi_{T}^{M}\left(\boldsymbol{\omega}_{2}\right)(\boldsymbol{U}+\varepsilon \boldsymbol{V} ; \boldsymbol{\Gamma})-\Phi_{T}^{M}\left(\boldsymbol{\omega}_{1}\right)(\boldsymbol{U} ; \boldsymbol{\Gamma})}{\varepsilon} \\
& \approx \frac{\Phi_{T}^{M}\left(\boldsymbol{\omega}_{2}\right)(\boldsymbol{U} ; \boldsymbol{\Gamma})+\varepsilon D \Phi_{T}^{M}\left(\boldsymbol{\omega}_{2}\right)(\boldsymbol{U} ; \boldsymbol{\Gamma}) \boldsymbol{V}-\Phi_{T}^{M}\left(\boldsymbol{\omega}_{1}\right)(\boldsymbol{U} ; \boldsymbol{\Gamma})}{\varepsilon}
\end{aligned}
$$

with $\varepsilon \ll 1$ will result in an $\mathcal{O}\left(1 /\left(\varepsilon^{2} M\right)\right)$ variance.

Consequently, the variance of $D \Phi_{T}^{M}(\boldsymbol{U} ; \boldsymbol{\Gamma}) \boldsymbol{V}$ will grow unboundedly as $\varepsilon$ tends to zero. One should therefore aim to use the same random numbers twice, both with the unperturbed and perturbed initial conditions. A method to enforce the use of the same random numbers is proposed in section 4.3 .

4.2. Lifting and restriction. In this section, we describe two lifting operators, as well as their corresponding restriction operator. For both approaches, the microscopic parameters are initialized by generating i.i.d. samples for each agent in each realization from the governing probability distributions (2.1). The difference between the two lifting (restriction) operators is limited to the initialization of the microscopic state. We emphasize as well that, for each realization, the microscopic state is initialized independently of the parameter values.

4.2.1. Simple lifting and restriction. Let us first describe a simple approach. We are given a macroscopic state $\boldsymbol{U}=\left(U_{n}\right)_{n=1}^{N}$, and we want to generate $M$ realizations of $N$ agents, consistently with that macroscopic state. To create these microscopic realizations $\boldsymbol{B}=\left[\boldsymbol{u}^{m}\right]_{m=1}^{M}$, with $\boldsymbol{u}^{m}=\left(u_{n}^{m}\right)_{n=1}^{N}$, we can sample, at each $x_{n}$, the Bernoulli distribution with mean $U_{n}$, that is,

$$
u_{n}^{m} \sim \mathcal{B}\left(u ; U_{n}\right) \Longleftrightarrow\left\{\begin{array}{l}
\operatorname{Pr}\left(u_{n}^{m}=1\right)=U_{n}, \\
\operatorname{Pr}\left(u_{n}^{m}=0\right)=1-U_{n},
\end{array} \quad m=1, \ldots, M .\right.
$$

Combining this sampling of the microscopic state with a sampling procedure for the microscopic parameters of the individual agents, we obtain a lifting operator $\mathcal{L}(\boldsymbol{\omega})$ of the type (4.3). The corresponding restriction operator is then given by taking the empirical average over the set of $M$ realizations,

$$
\mathcal{R}: \mathbb{B}^{N \times M} \longrightarrow \mathbb{R}^{N}, \quad \boldsymbol{B} \longmapsto \boldsymbol{U}=\frac{1}{M} \sum_{m=1}^{M} \boldsymbol{u}^{m} .
$$

The simple lifting and restriction operators defined above cannot satisfy the consistency condition (4.5) for an arbitrary value of $\boldsymbol{U}(t)$, since the restriction can only map onto $\mathbb{Q}_{M}^{N}=$ $\mathbb{Q}_{M} \times \cdots \times \mathbb{Q}_{M}$ instead of onto $\mathbb{R}^{N}$; i.e., only integer fractions of $M$ can be represented. The incurred discrepancy is essentially a sampling error, since the sampling procedure outlined above only ensures (4.5) in the limit $M \rightarrow \infty$. Indeed, when $(\boldsymbol{B}, \boldsymbol{A})=\mathcal{L}(\boldsymbol{U}, \boldsymbol{\Gamma})$, with $\boldsymbol{B}=$ $\left[\boldsymbol{u}^{m}\right]_{m=1}^{M}$, then

$$
\lim _{M \rightarrow \infty} \frac{1}{M} \sum_{m=1}^{M} \boldsymbol{u}^{m}=\boldsymbol{U}
$$

Copyright (c) by SIAM. Unauthorized reproduction of this article is prohibited. 
4.2.2. Weighted lifting and restriction. The main idea of the present paper, which is key to all the numerical methods that follow, is the introduction of a new restriction operator that replaces the empirical average (4.13) by a weighted average of the form

$$
\mathcal{R}_{\mathrm{w}}(\boldsymbol{w}): \mathbb{B}^{N \times M} \longrightarrow \mathbb{R}^{N}, \quad \boldsymbol{B} \longmapsto \boldsymbol{U}=\frac{1}{M} \sum_{m=1}^{M} w^{m} \boldsymbol{u}^{m}
$$

in which $\boldsymbol{w}=\left[w^{m}\right]_{m=1}^{M} \in \mathbb{R}^{M}$ is a vector of weights satisfying

$$
\frac{1}{M} \sum_{m=1}^{M} w^{m}=1
$$

The restriction operator $\mathcal{R}_{\mathrm{w}}$ is specified completely only once the weights $\boldsymbol{w}$ are known; they will be selected such that the constraint (4.5) is satisfied exactly, which implies that the restriction operator will depend on the specific realizations $\boldsymbol{u}^{m}$ that were generated during the lifting.

Before outlining the procedure, let us highlight the rationale behind the introduction of the weighted average. As noted in Remark 2.5, the probability distribution $P(\boldsymbol{u}, t)$ can be discretized according to two guiding principles: (i) deterministically, that is, we consider every possible realization and attach to it a probability weight expressing how likely the realization is to occur, which results in (2.11); or (ii) stochastically, that is, we sample a finite number of realizations from the corresponding probability distributions, resulting in the estimate (2.12). Option (i) is infeasible because it requires considering $M=2^{N}$ realizations (many of which will be extremely unlikely), while option (ii) will contain a sampling error such that the identity (4.5) is violated. Introducing the weighted restriction (4.15) can then be seen as a hybrid approach that allows satisfying (4.5) with a limited number of realizations $M \ll 2^{N}$; the condition (4.16) ensures that the weight $w^{m}$, attached to the realization $\boldsymbol{u}^{m}$, can be interpreted as the probability of obtaining that realization out of all the realizations in the sample. This interpretation also imposes the condition that all the weights be positive. We shall see that there is an interplay between the creation of the $M$ realizations and the computation of the corresponding weights for the restriction.

A possible way to compute weights is the following: first, we generate $M^{\prime}$ realizations $\boldsymbol{u}^{m}$ according to the naive procedure (4.12); since we know that this procedure yields the desired result as $M^{\prime}$ tends to infinity, it seems reasonable to attach weights that are as close to 1 as possible, while satisfying the identity (4.5) as well as the constraint (4.16). As will become clear further on, this procedure will allow for optimization problems that are either infeasible (with no possible solutions) or ill-posed (with infinitely many possible solutions). To see this,

Copyright $\odot$ by SIAM. Unauthorized reproduction of this article is prohibited. 
we formulate the following least squares problem:

$$
\begin{aligned}
& \boldsymbol{w}=\arg \min \frac{1}{2} \sum_{m=1}^{M^{\prime}}\left(w^{m}-1\right)^{2}, \\
& \frac{1}{M^{\prime}} \sum_{m=1}^{M^{\prime}} w^{m} \boldsymbol{u}^{m}=\boldsymbol{U}, \\
& \frac{1}{M^{\prime}} \sum_{m=1}^{M^{\prime}} w^{m}=1, \\
& w^{m} \geq 0, \quad 1 \leq m \leq M^{\prime} .
\end{aligned}
$$

We recall here a basic result in minimization problems [29, 46].

Lemma 4.3. Let us consider the equality-constrained quadratic minimization problem

$$
\begin{aligned}
& \boldsymbol{w}=\arg \min \frac{1}{2} \boldsymbol{w}^{T} \boldsymbol{w}-\boldsymbol{g}^{T} \boldsymbol{w}, \\
& \boldsymbol{C} \boldsymbol{w}=\boldsymbol{b}
\end{aligned}
$$

where $\boldsymbol{w}, \boldsymbol{g} \in \mathbb{R}^{M}, \boldsymbol{b} \in \mathbb{R}^{N}$ and where $\boldsymbol{C} \in \mathbb{R}^{N \times M}$, with $N<M$, is a constraint matrix with full rank; then

$$
\left[\begin{array}{cc}
\boldsymbol{I} & \boldsymbol{C}^{T} \\
\boldsymbol{C} & 0
\end{array}\right]\left[\begin{array}{l}
\boldsymbol{w} \\
\boldsymbol{\lambda}
\end{array}\right]=\left[\begin{array}{l}
\boldsymbol{g} \\
\boldsymbol{b}
\end{array}\right]
$$

where $\boldsymbol{\lambda} \in \mathbb{R}^{N}$ is the associated Lagrange multiplier. The linear system (4.21) has a unique solution.

Let us now consider the difficulties that may lead to a rank-deficient constraint matrix $\boldsymbol{C}$ :

1. The sampling procedure (4.12) can yield multiple identical realizations of the microscopic state (identical columns in the constraint matrix). For instance, this may happen with high probability if the macroscopic state $\boldsymbol{U}$ is close to 0 or 1 for all agents, such that all realizations consist of almost all 0 or all 1 .

2. The sampling procedure can also yield repeated rows in the constraint matrix when two agents $\left(n_{1}\right.$ and $\left.n_{2}\right)$ have an identical state in each of the realizations, that is, $u_{n_{1}}^{m}=u_{n_{2}}^{m}$ for all $m$. This also happens with high probability if the macroscopic state $U_{n}$ is close to 0 or 1 for two or more agents.

3 . For a given agent $n$, one might find that all realizations have the same value ( 0 or 1$)$. When $U_{n}$ is not identically 0 or 1 , this leads to an infeasible constraint; again, this situation is likely to occur when the macroscopic state $U_{n}$ for some agent $n$ is close to 0 or 1 .

To circumvent these problems, we will discard duplicate realizations during the computation of the weights and extend the sample set with artificially created samples. To minimize perturbations with respect to the underlying probability distributions, the target weights $\boldsymbol{g}$ will be adjusted accordingly.

To be specific, we circumvent the first problem as follows. We denote by $\boldsymbol{B}^{\prime}$ the ensemble of $M^{\prime}$ realizations that were generated with the procedure (4.12) and write this ensemble in

Copyright (C) by SIAM. Unauthorized reproduction of this article is prohibited. 
a different representation $(\tilde{\boldsymbol{B}}, \boldsymbol{g})$ where we only retain unique realizations, as well as their cardinality in the ensemble $\boldsymbol{B}^{\prime}$,

$$
\tilde{\boldsymbol{B}}=\left[\boldsymbol{u}^{m}\right]_{m=1}^{\tilde{M}}, \quad \boldsymbol{g}=\left[g^{m}\right]_{m=1}^{\tilde{M}},
$$

where

$$
g^{m}=\#\left\{\boldsymbol{u}^{m} \in \boldsymbol{B}^{\prime}\right\} \quad \text { for all } m \text { such that } \boldsymbol{u}^{m} \in \tilde{\boldsymbol{B}} .
$$

We note that, by definition, we have $\sum_{m=1}^{\tilde{M}} g^{m}=M^{\prime}$. During optimization, we will then compute a weight for each single realization that is close to $g^{m}$ (see what follows) to take into account the fact that each realization appeared $g^{m}$ times in our original sampling. Note that afterward we retain all individual realizations, since they will have different values for the microscopic parameters over which we want to average.

To circumvent the second and third problems, we create artificial realizations in the lifting step that are unlikely to be obtained by the naive sampling procedure (4.12), and we assign to them a target weight of 0 to minimize artifacts in the resulting probability distributions. First, we scan the new constraint matrix and search for duplicate rows. For each repeated row $n_{\mathrm{r}}$, we add a realization as follows:

$$
u_{n}= \begin{cases}1 & \text { if } n=n_{\mathrm{r}} \\ 0 & \text { otherwise }\end{cases}
$$

Then, we check if there exists a row $n_{0}$ of 0 's or a row $n_{1}$ of 1 's and if so add the following realizations, respectively:

$$
u_{n}=\left\{\begin{array}{ll}
1 & \text { if } n=n_{0}, \\
0 & \text { otherwise }
\end{array} \quad u_{n}= \begin{cases}0 & \text { if } n=n_{1}, \\
1 & \text { otherwise }\end{cases}\right.
$$

We collect all additional realizations (4.24)-(4.25) in the set $\boldsymbol{B}^{\prime \prime}$ and compute the cardinality as follows:

$$
g^{m}=0 \quad \text { for all } m \text { such that } \boldsymbol{u}^{m} \in \boldsymbol{B}^{\prime \prime}
$$

indicating that those realizations appear with cardinality 0 in the original sampling according to procedure (4.12) and have only been added to regularize the constraint matrix.

A weighted lifting operator $\mathcal{L}_{\mathrm{w}}(\boldsymbol{\omega})$ is then given as the set of $M=M^{\prime}+M^{\prime \prime}$ realizations $\boldsymbol{B}=\boldsymbol{B}^{\prime} \cup \boldsymbol{B}^{\prime \prime}$, along with the correspondingly sampled microscopic parameter values $\boldsymbol{A}$, which together form an operator of the type (4.3). The weights $\boldsymbol{w} \in \mathbb{R}^{M}$ (with $M=M^{\prime}+M^{\prime \prime}$ ) that will be used in the restriction $\mathcal{R}_{\mathrm{w}}$ are such that both (4.15) and (4.16) are satisfied, and such that the natural sampling frequencies, as exemplified by the counters $g^{m}$, are matched as closely as possible. We first compute weights $\tilde{\boldsymbol{w}} \in \mathbb{R}^{\tilde{M}+M^{\prime \prime}}$ for all elements of $\tilde{\boldsymbol{B}} \cup \boldsymbol{B}^{\prime \prime}$ by 
solving the regularized constrained minimization problem

$$
\begin{aligned}
& \tilde{\boldsymbol{w}}=\arg \min \frac{1}{2} \sum_{m=1}^{\tilde{M}+M^{\prime \prime}}\left(\tilde{w}^{m}-\frac{M}{M^{\prime}} g^{m}\right)^{2}, \\
& \frac{1}{M} \sum_{m=1}^{\tilde{M}+M^{\prime \prime}} \tilde{w}^{m} \boldsymbol{u}^{m}=\boldsymbol{U}, \\
& \frac{1}{M} \sum_{m=1}^{\tilde{M}+M^{\prime \prime}} \tilde{w}^{m}=1, \\
& \tilde{w}_{m} \geq 0, \quad 1 \leq m \leq \tilde{M}+M^{\prime \prime} .
\end{aligned}
$$

In the system above, we conventionally assumed that $\boldsymbol{u}^{m} \in \tilde{\boldsymbol{B}}$ when $1 \leq m \leq \tilde{M}$ and $\boldsymbol{u}^{m} \in \boldsymbol{B}^{\prime \prime}$ when $\tilde{M}<m \leq \tilde{M}+M^{\prime \prime}$. The choice of the goal function (4.27) ensures that constraints (4.28) and (4.29) are not affected by the presence of additional realizations with weights that are identically zero, that is, $w^{m}=\left(M / M^{\prime}\right) g^{m}$ is a solution that satisfies (4.29).

We then transform the weights $\tilde{\boldsymbol{w}} \in \mathbb{R}^{\tilde{M}+M^{\prime \prime}}$ back to weights $\boldsymbol{w} \in \mathbb{R}^{M}$ for the $M=M^{\prime}+M^{\prime \prime}$ realizations in $\boldsymbol{B}=\boldsymbol{B}^{\prime} \cup \boldsymbol{B}^{\prime \prime}$. This is done by selecting, for each element $\boldsymbol{u}^{m} \in \boldsymbol{B}^{\prime}$, the (unique) index $\tilde{m}^{*}$ such that $\boldsymbol{u}^{m}=\boldsymbol{u}^{\tilde{m}^{*}}$ with $\boldsymbol{u}^{\tilde{m}^{*}} \in \tilde{\boldsymbol{B}}$, and setting $w^{m}=\tilde{w}^{m^{*}} / g^{m^{*}}$ for $1 \leq m \leq \tilde{M}$.

Remark 4.4 (effect of regularization on probability distributions of the microscopic states). $A$ natural question arises as to whether the regularization procedure proposed above has an impact on the probability distributions of the microscopic states. With the regularization, we amend the lifted realizations in two ways. First, we remove identical realizations from the constraint matrix, and we assign to the corresponding weight a higher target (the vector $\boldsymbol{g}$ in (4.27) contains the cardinality of the unique realizations in $\left.\boldsymbol{B}^{\prime}\right)$ : with this procedure we do not alter the underlying probability distribution of the microscopic states, in that realizations that have been removed will have a correspondingly higher weight. Second, we add artificial realizations, which in principle create a bias in the underlying microscopic distribution: for this bias not to affect the outcome of our computations, the associated weights should be vanishingly small; hence we prescribe for them a target equal to 0 (again via the vector $\boldsymbol{g}$ ), and we expect that these weights tend to 0 as $M \rightarrow \infty$.

Remark 4.5 (numerical solution of the minimization problem). We solve (4.27)-(4.29) using a single Cholesky factorization [46]. Algorithms based on the conjugate gradient method can also be employed for large equality-constrained quadratic problems [29]. In our computation, we do not explicitly require (4.30): positivity of the weights is assessed in a postprocessing step and used to determine whether enough realizations were taken (we increase $M$ until all weights are positive). It is also possible (albeit more expensive) to include the inequality constraints (4.30) and use iterative methods to solve the minimization problem [46].

4.3. Variance-reduced Jacobian-vector products. Let us now discuss the Jacobian-vector multiplication that was introduced in (4.11). As indicated before, a problem with using (4.11) directly is the presence of numerical noise, which should be addressed by using the same random numbers in both the unperturbed and perturbed simulations. To achieve this, we use the same realizations, microscopic parameters, and random time paths in both the perturbed and

Copyright (C) by SIAM. Unauthorized reproduction of this article is prohibited. 

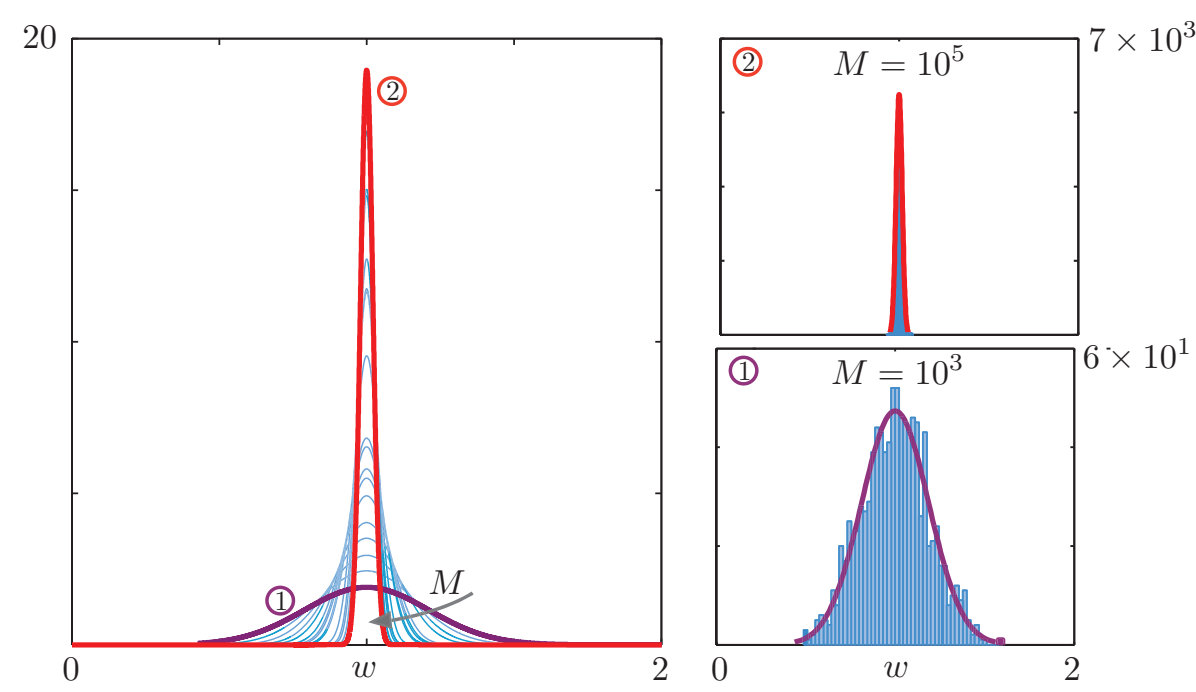

Figure 6. Distribution of weights as a function of the total number of realizations $M$. Weights are obtained by lifting a mixed state and solving the corresponding minimization problem for values of $M$ ranging between $10^{3}$ (curve 1 , magenta) and $10^{5}$ (curve 2 , red). The resulting data is fitted to a Gaussian distribution. As $M$ increases, the weights are sharply distributed around 1. Parameters are as in E1 in Table 1.

unperturbed coarse time-steppers; the only difference is in the computation of the weights. For the perturbed coarse time-stepper, we replace the constrained optimization problem for the weights by

$$
\begin{aligned}
& \tilde{\boldsymbol{w}}_{\varepsilon}=\arg \min \frac{1}{2} \sum_{m=1}^{\tilde{M}+M^{\prime \prime}}\left(\tilde{w}_{\varepsilon}^{m}-\frac{M}{M^{\prime}} g^{m}\right)^{2}, \\
& \frac{1}{M} \sum_{m=1}^{\tilde{M}+M^{\prime \prime}} \tilde{w}_{\varepsilon}^{m} \boldsymbol{u}^{m}=\boldsymbol{U}+\varepsilon \boldsymbol{V}, \\
& \frac{1}{M} \sum_{m=1}^{\tilde{M}+M^{\prime \prime}} \tilde{w}_{\varepsilon}^{m}=1, \\
& \tilde{w}_{\varepsilon}^{m} \geq 0, \quad 1 \leq m \leq M .
\end{aligned}
$$

Note that only the constraint (4.32) has changed with respect to the unperturbed optimization problem (see (4.28)). Since the solution of the optimization problem depends continuously and differentiably on the right-hand side of the constraints, small perturbations on the right-hand side of (4.32) lead to small perturbations in weights. Furthermore, since we are using the same microscopic realizations $\boldsymbol{u}^{m}$ in the constraints of the perturbed and unperturbed minimization problems, we have effectively imposed $\boldsymbol{\omega}_{1}=\boldsymbol{\omega}_{2}$ in the finite difference formula (4.11); hence the variance of $D \boldsymbol{\Phi}\left(\boldsymbol{\omega}_{1}\right)(\boldsymbol{U}) \boldsymbol{V}$ is bounded and of $\mathcal{O}(1 / M)$.

In the limit of infinitely many realizations (where all weights converge to 1 ), the presented procedure converges to the exact Jacobian-vector product. For finite values of $M$, there will be noise in the Jacobian-vector product as a result of the random selection of a subset of all

Copyright ( $\odot$ by SIAM. Unauthorized reproduction of this article is prohibited. 


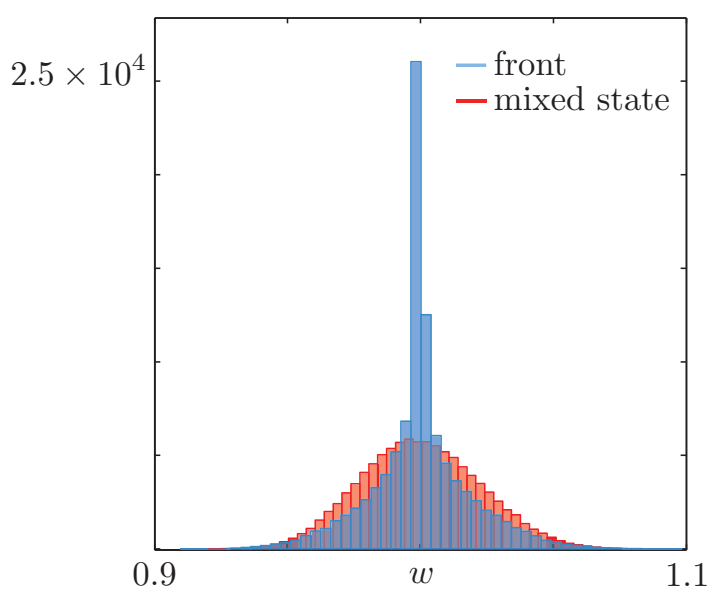

Figure 7. Distribution of weights with $M=10^{5}$ realizations for two different macroscopic steady states: A mixed state (red) and a front (blue). Both distributions are sharply peaked around one, albeit the distribution for fronts is not Gaussian. Parameters are as in E1 (mixed state) and E3 (front) in Table 1.

possible realizations. The procedure only prevents noise blow-up that would arise if a different selection of realizations were considered for the perturbed and unperturbed coarse time step.

5. Numerical properties of the equation-free method. In this section we show a series of numerical tests that highlight the numerical properties of the weighted lifting and lead to an appropriate calibration of the Newton-GMRES solver. For our tests we used a population of either 40 or 400 agents, a number of realizations varying between $10^{3}$ and $10^{5}$, and different types of macroscopic steady states. Here and henceforth we will denote by locked-in states homogeneous macroscopic states with $U_{n} \approx 0$ or $U_{n} \approx 1$ for all $n$, by mixed states solutions with $U_{n} \approx 0.5$ for all $n$, and by fronts solutions that connect two locally locked-in states. For these solutions, which were previously found via direct numerical simulations in Figures 1-4, we use parameters of E1-E3 in Table 1. Note that, when computing fronts, we effectively restrict our computations to one-dimensional lattices (which develop along the $x$ direction) and discard the $y$-coordinate of the lattice. We stress that the numerical procedure presented here is unchanged in the case of two-dimensional patterns. Unless otherwise stated, we set a time horizon $T=20$ for the coarse time-stepper.

5.1. Convergence of the weights. In our first numerical experiment, we fix $N=40$, lift macroscopic steady states with the weighted operator $\mathcal{L}_{\mathrm{w}}$, and plot the weight distribution as a function of the number of realizations $M$. By construction (see section 4.2), we expect weights to be sharply distributed around 1 as $M$ tends to infinity. In Figure 6 , we lift a mixed state for various $M$ and observe that the weight distribution is well approximated by a Gaussian and tends to a Dirac distribution as $M \rightarrow \infty$.

Similar results are obtained (not shown) for locked-in states and fronts. However, we note that weights distributions associated with these states are not necessarily Gaussian, as shown in Figure 7. We point out that for such macroscopic states, many weights are assigned a goal equal to 0 , according to (4.26). It is not surprising that the distributions for these states, for which $M^{\prime \prime} \gg 1$, are different from those associated with a mixed state, for which $M^{\prime \prime} \approx 0$ (see

Copyright (c) by SIAM. Unauthorized reproduction of this article is prohibited. 


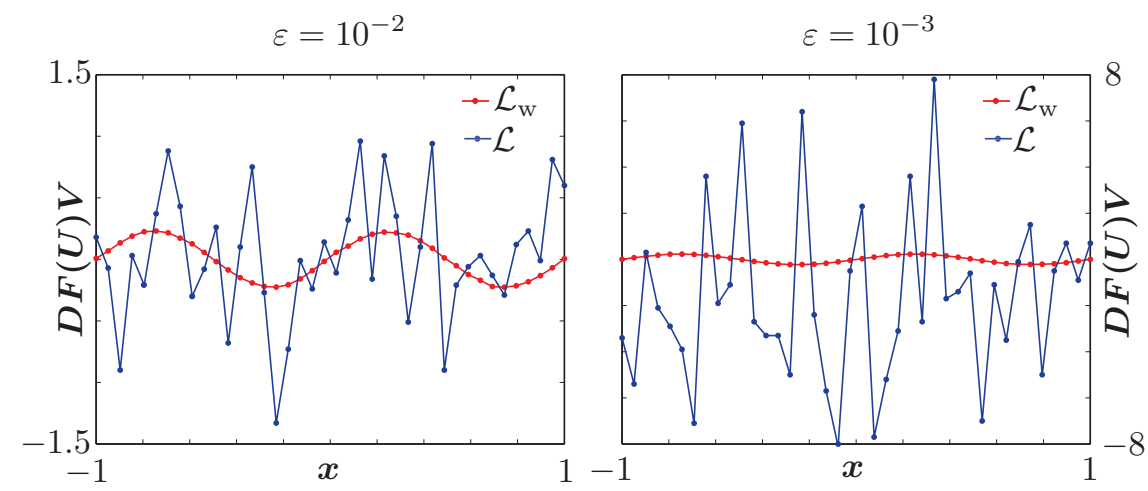

Figure 8. Jacobian-vector product $\boldsymbol{D} \boldsymbol{F}(\boldsymbol{U}) \boldsymbol{V}$ of $\boldsymbol{F}(\boldsymbol{U})=\boldsymbol{U}-\boldsymbol{\Phi}_{T}^{M}(\boldsymbol{U})$. We use a macroscopic front solution $\boldsymbol{U}$ and a sinusoidal perturbation $\boldsymbol{V}$ with $\|\boldsymbol{V}\|_{2}=1$. The unweighted lifting produces inaccurate Jacobian-vector product evaluations whose norm becomes unbounded as we decrease the relative size of the perturbation $\varepsilon$. On the other hand, weighted lifting preserves the structure of the perturbation. For this experiment we used $N=40$, $M=10^{4}$, whereas all other parameters are chosen as in E3 of Table 1.

also Remark 4.4).

5.2. Convergence of the Jacobian-vector product. We test the numerical properties of weighted Jacobian-vector products with a second numerical experiment. We select a region of parameter space in which a stable macroscopic front $\boldsymbol{U}$ is observed (corresponding to E3 in Table 1) and compute a single evaluation of the Jacobian-vector product $\boldsymbol{D} \boldsymbol{F}(\boldsymbol{U}) \boldsymbol{V}$, where $\boldsymbol{F}$ is given by (4.9), $\boldsymbol{D} \boldsymbol{\Phi}$ is estimated by (4.11), and $\boldsymbol{V}$ has unit norm and a sinusoidal profile in $x$. If $\boldsymbol{\Phi}(\boldsymbol{U}+\epsilon \boldsymbol{V})$ and $\boldsymbol{\Phi}(\boldsymbol{U})$ are calculated using two independent function evaluations, the Jacobian-vector product is severely affected by noise and completely loses the structure of the perturbation $\boldsymbol{V}$ (blue lines in Figure 8). Furthermore, this effect is greatly amplified as we decrease $\varepsilon$, as the Jacobian-vector product becomes unbounded.

On the other hand, using weighted operators and the variance-reduced Jacobian-vector product outlined in section 4.3, we maintain the structure of the perturbation, and the Jacobian-vector product varies smoothly as a function of $x$. A further confirmation is found in Figure 9, where we plot the 2-norm of $\boldsymbol{F}(\boldsymbol{U}+\varepsilon \boldsymbol{V})-\boldsymbol{F}(\boldsymbol{U})$ as a function of $\varepsilon$. In particular, we seek the minimum number of realizations required to obtain smooth Jacobian evaluations, that is, an $\mathcal{O}(\varepsilon)$ curve: if $N=40$, then 100 weighted realizations are sufficient to obtain a smooth Jacobian evaluation, whereas 10000 unweighted realizations are still affected by noise (left panel of Figure 9). If we increase the system size to $N=400$, then 1000 weighted realizations are sufficient to observe an $\mathcal{O}(\varepsilon)$ curve. The experiments in Figure 9 show that weights are effective with $M=\mathcal{O}(N)$ realizations.

5.3. Convergence of GMRES iterations. The next step toward the construction of our Newton-Krylov solver is the solution of the linear system associated with the Jacobian $\boldsymbol{D F}$ of $\boldsymbol{F}$. We use GMRES to iteratively solve the system $\boldsymbol{D} \boldsymbol{F}(\boldsymbol{U}) \boldsymbol{V}=-\boldsymbol{F}(\boldsymbol{U})$, where $\boldsymbol{U}$ is a mixed state and $\boldsymbol{D F}$ is computed using weighted operators and variance-reduced Jacobian-vector products. In Figure 10 we show convergence plots for the GMRES solver for various numbers of realizations and system sizes. In our computations we choose $\varepsilon=10^{-5}$ for the finite difference

Copyright (c) by SIAM. Unauthorized reproduction of this article is prohibited. 

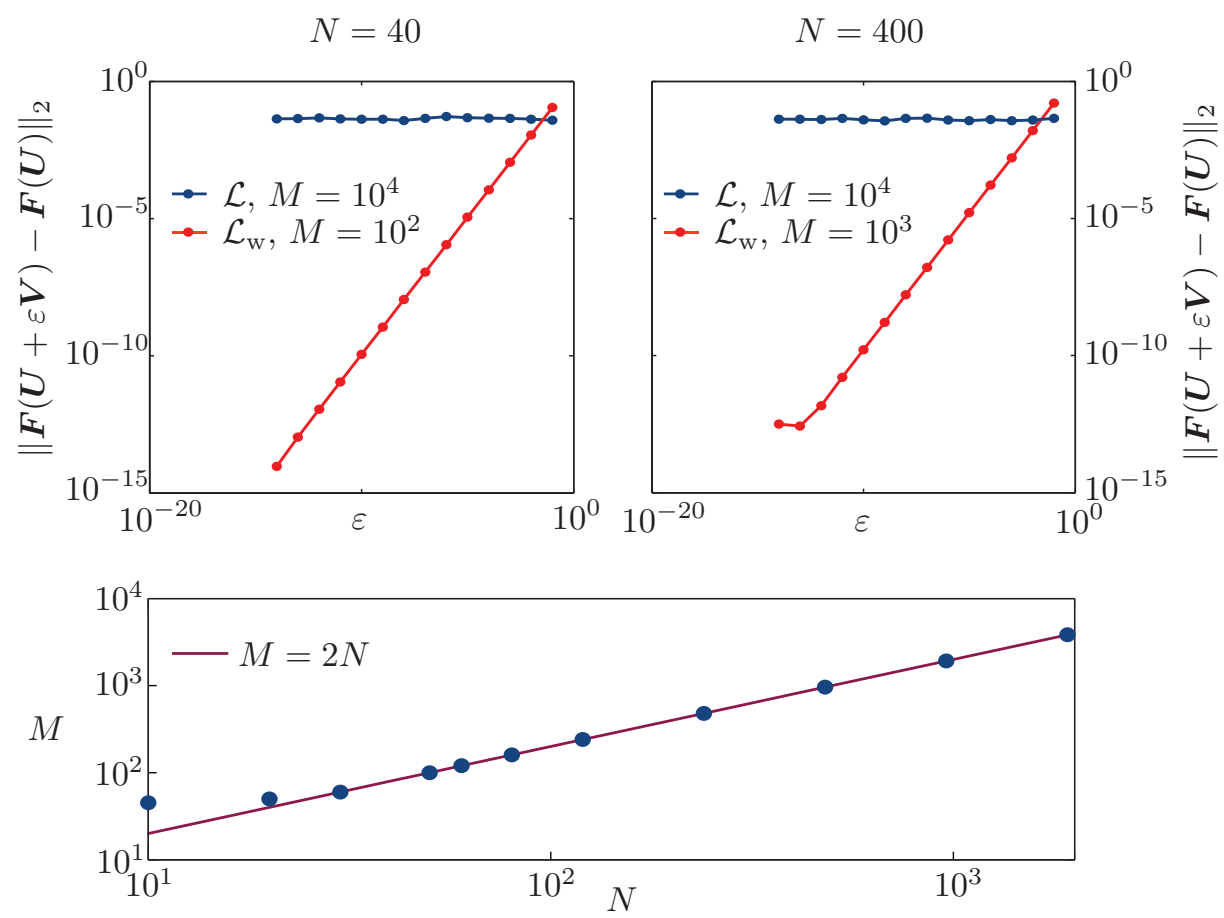

Figure 9. For a front $\boldsymbol{U}$ and a unit-norm random vector $\boldsymbol{V}$, we compute the 2-norm of $\boldsymbol{F}(\boldsymbol{U}+\varepsilon \boldsymbol{V})-\boldsymbol{F}(\boldsymbol{U})$ as a function of $\varepsilon$, using weighted and unweighted lifting operators, for $\boldsymbol{F}(\boldsymbol{U})=\boldsymbol{U}-\Phi_{T}^{M}(\boldsymbol{U})$. Top left: If we set $N=40$ and use $10^{4}$ unweighted realizations, noise affects the evaluation of the Jacobian action, whereas $10^{2}$ weighted realizations are sufficient to obtain an $\mathcal{O}(\varepsilon)$ curve. Top right: The experiment is repeated for $N=400$. Bottom: We repeat the computations in the top panels for various values of $N$ and show the number of weighted realizations which consistently give an $\mathcal{O}(\varepsilon)$ curve in the Jacobian evaluation. The experiment shows that weights become effective with $M=\mathcal{O}(N)$ realizations.

approximation of the Jacobian, set model parameters as in E3 in Table 1, and employ the built-in MATLAB function gmres with restart $=20$, tol $=10^{-5}$, maxit $=20$. As we can see, the linear problems are well behaved. As expected, the linear iterations necessary to obtain convergence decrease as we increase the number of realizations but increase with the system size.

5.4. Convergence of Newton-GMRES. We now proceed to the computation of a single front, for fixed values of the control parameters, using the Newton-GMRES method. We start our iterations with a mixed state, $U_{n}=0.5$ for all $n$, and converge to a front, whose profile for various values of the number of realizations is shown in Figure 11. In panels (a) and (c) of Figure 12, we show convergence plots of the Newton-GMRES solver for $N=40$ and $N=400$, with various numbers of realizations. In these plots we scale the residual by $\sqrt{N}$ so as to compare performances with varying system sizes. The Newton steps are built around the linear solves described in section 5.3, and each Newton update is damped by a constant factor 0.5 .

In the low-dimensional case, $N=40$, the solver achieves convergence in fewer than 4 iterations, and then residuals plateau and begin to oscillate, as expected (panel (a) of Figure 12).

Copyright (C) by SIAM. Unauthorized reproduction of this article is prohibited. 

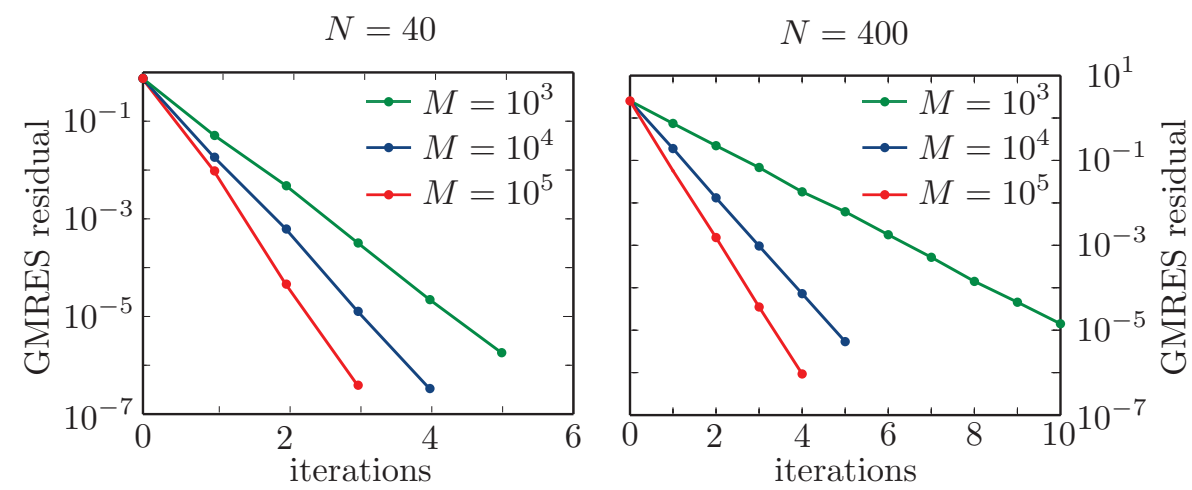

Figure 10. Convergence of GMRES iterations for the problem $\boldsymbol{D} \boldsymbol{F}(\boldsymbol{U}) \boldsymbol{V}=-\boldsymbol{F}(\boldsymbol{U})$, where $\boldsymbol{U}$ is a macroscopic front and $\boldsymbol{D} \boldsymbol{F}(\boldsymbol{U})=\boldsymbol{I}-\boldsymbol{D} \boldsymbol{\Phi}_{T}^{M}(\boldsymbol{U})$ for different numbers of agents $N$ and numbers of realizations $M$. Jacobian actions are computed with weighted lifting and $\varepsilon=10^{-5}$.
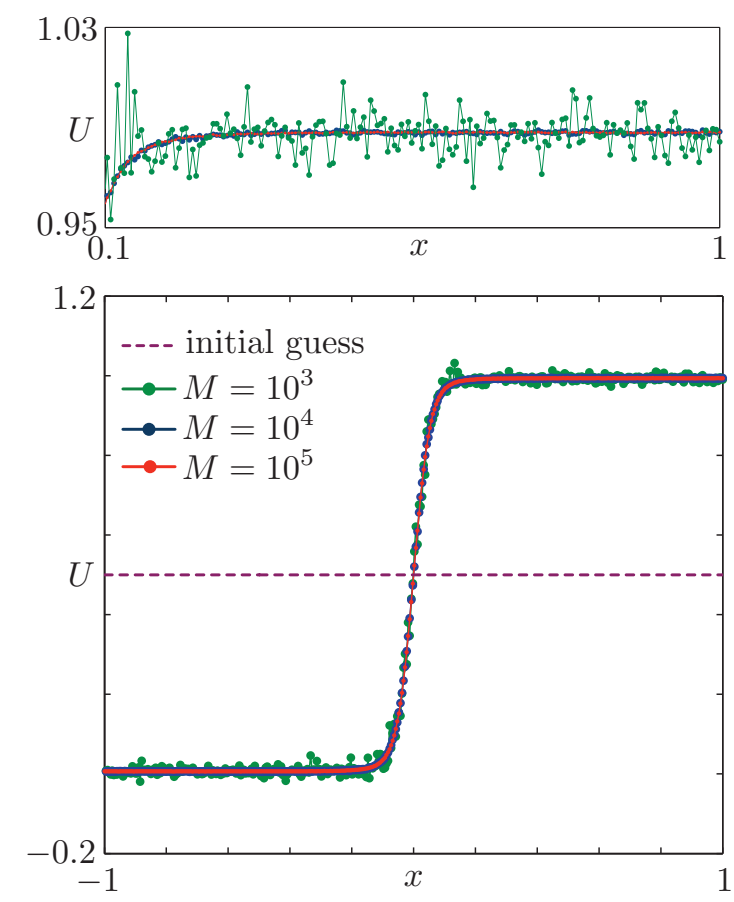

Figure 11. Macroscopic fronts computed with the Newton-GMRES solver (see also Figures 12(c) and $12(\mathrm{~d})$ ). The initial guess for all cases is a mixed state $U(x) \equiv 0.5$. The inset on top shows that the noise in the macroscopic profile is controlled by increasing the number of realizations.

The onset of these oscillations is an indication of the best tolerance that we can achieve with the nonlinear solver for a fixed number of realizations: such tolerance is of $\mathcal{O}(1 / \sqrt{M})$, as is shown in panel (b). In the high-dimensional case, $N=400$, a similar scenario occurs, though more iterations are needed to achieve convergence. We point out that the experiment of Figure 12 represents a severe test for the nonlinear solver in that we have chosen a poor initial guess (we start from a mixed state to obtain a front). During continuation, initial guesses are

Copyright $\odot$ by SIAM. Unauthorized reproduction of this article is prohibited. 
(a)

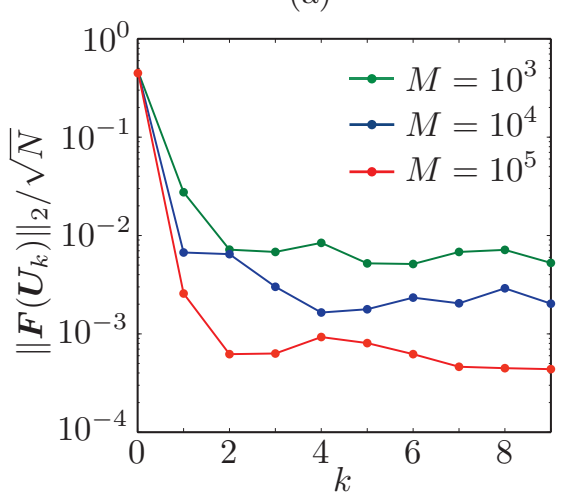

(c)

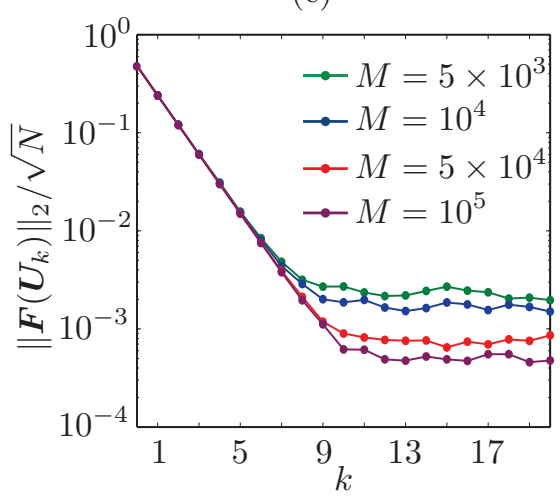

(b)

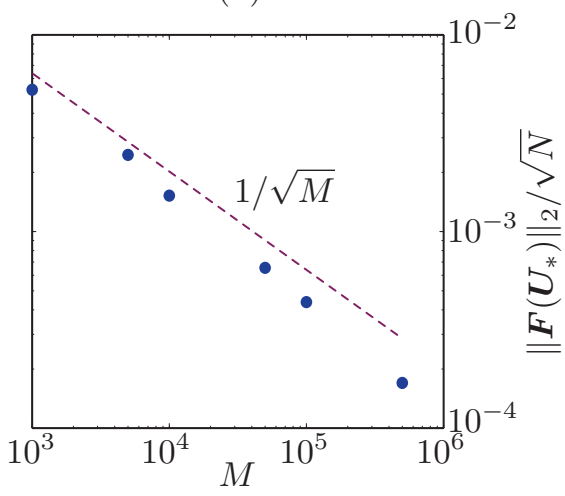

(d)

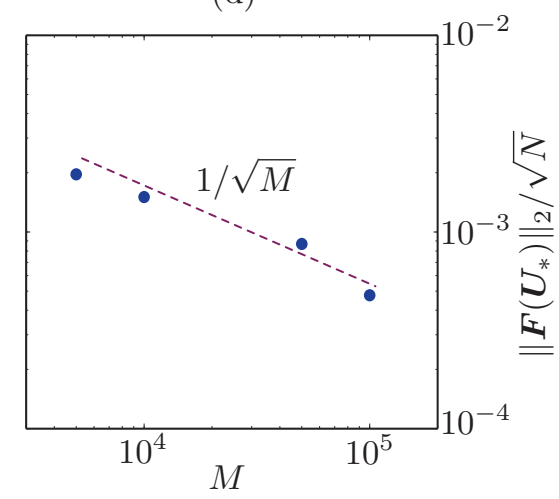

Figure 12. Convergence of the Newton-GMRES solver to compute a macroscopic front solution. The initial guess is a mixed state $U(x) \equiv 0.5$, and the Newton steps are damped with a constant factor 0.5. Parameters are as in $\mathrm{E} 1$ of Table 1, with the exception of $\alpha=0.7$. (a) Convergence for various values of the number of realizations $M$, for $N=40$. (b) The best achieved tolerance in the experiment of (a) is an $\mathcal{O}(1 / \sqrt{M})$. (c) $-(\mathrm{d})$ The computations of (a) and (b) are repeated with $N=400$. Solution profiles for (c) and (d) are shown in Figure 11.

provided by a tangent or secant predictor step, resulting in much faster convergence. We also remark that the convergence of the nonlinear solver is linear, as expected, since we are using damped Newton updates.

6. Bifurcation study of vendor lock-in model. In this section we present the results of coarse-grained numerical bifurcation analyses of the lock-in model. The bifurcation diagrams have been computed with a simple natural continuation method; that is, we start from a known solution to the steady-state problem (4.9), increment the continuation parameter, and solve a new problem using the previous solution as an initial guess. Even though this is not an optimal continuation strategy (as it does not allow one to go past folds with a single run), we employ it here mainly for its simplicity, keeping in mind that pseudoarclength continuation with tangent or secant prediction steps can easily be implemented. 

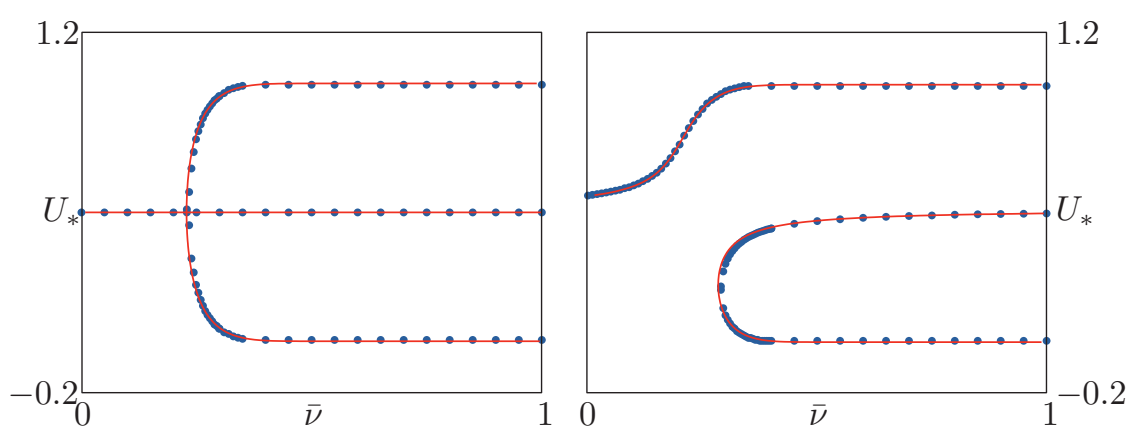

Figure 13. Coarse bifurcation diagram for homogeneous states. With blue dots we indicate the result of the equation-free continuation using weighted lifting operators. Red lines correspond to branches of fixed points of the approximate analytic evolution map (3.6), as in Figure 5. The bifurcation parameter is the average $\bar{\nu}$ of quality perception $q_{n}$, as reported in (2.2). Other parameters: $\bar{\mu}=0$ (left) and $\bar{\mu}=0.04$ (right), $\Delta \mu=0$, $\alpha=0, \bar{\xi}=0.236, \bar{\zeta}=0, \beta=10^{8}, N=400$. For the equation-free computations we use $M=10^{4}$ realizations and solve a 400-dimensional coarse system.
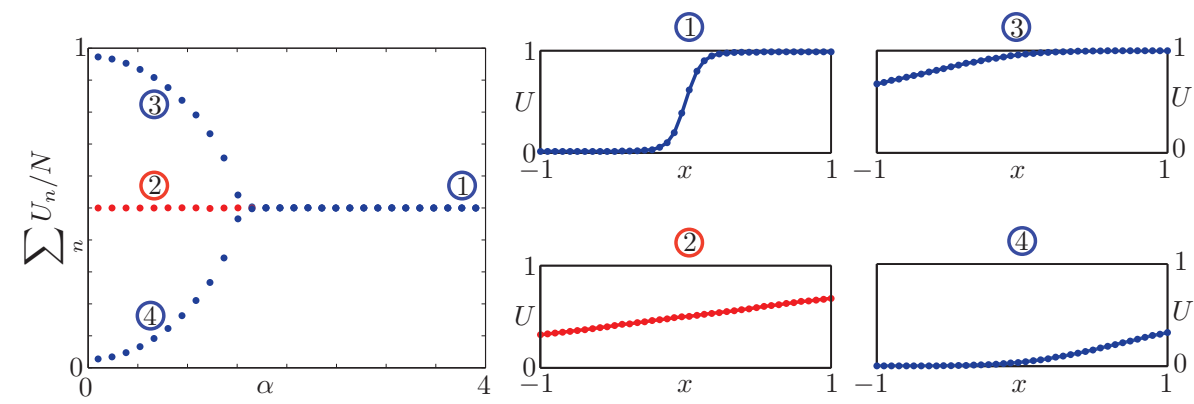

Figure 14. Coarse bifurcation diagram for fronts. The bifurcation parameter is the slope $\alpha$ of the profile of the average quality perception $q_{n}$, as reported in (2.2). For large $\alpha$ a stable macroscopic front is formed (solution 1). As $\alpha$ is decreased, the front destabilizes at a symmetry-breaking instability, generating partially locked-in states (solutions 3 and 4). A fixed continuation step of $\Delta \alpha=0.14$ has been used in the computations; other parameters are as in $\mathrm{E} 3$ of Table 1.

6.1. Continuation of homogeneous steady states. We compute branches of homogeneous states using weighted lifting operators for a population of $N=400$ agents. Since $N$ is large, we can compare our results with branches of fixed points of the approximate evolution map (3.6). In Figure 13 we compare a few branches, noting that discrepancies are due to the finite size of the system. We also point out that each point on the branch is the solution of a 400-dimensional coarse system: in principle we could have tracked the solution of a simple one-dimensional coarse system, since the solutions we are finding are homogeneous; however, this experiment provides a benchmark for our method and prepares us for the continuation of fronts.

6.2. Continuation of fronts as a function of $\alpha$. In Figure 14, we show a coarse bifurcation diagram of macroscopic fronts for a one-dimensional lattice with $N=40$ agents. We recall here that fronts are observed in the inhomogeneous lock-in model, for which

$$
q_{n} \sim \mathcal{N}\left(\bar{\mu}\left(x_{n}\right), \bar{\xi}\right), \quad \mu\left(x_{n}\right)=\bar{\mu}+\Delta \mu \tanh \left(\alpha x_{n}\right) .
$$

Copyright (c) by SIAM. Unauthorized reproduction of this article is prohibited. 


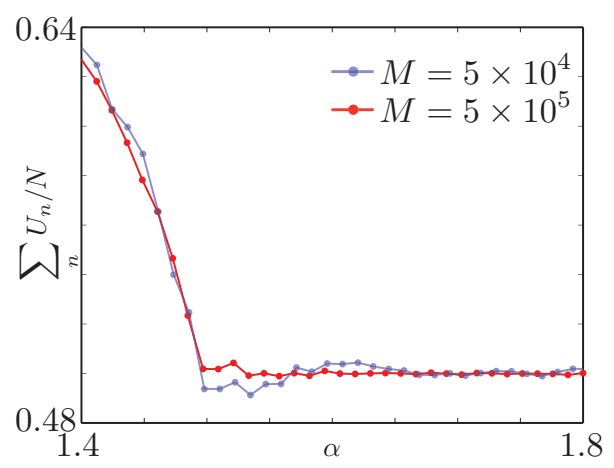

Figure 15. Effect of the number of realizations $M$ on the numerical continuation step size. We compute one of the branches in Figure 14 in the proximity of the symmetry-breaking bifurcation, with a much smaller continuation step, $\Delta \alpha=0.012$. The continuation with $5 \times 10^{4}$ realizations and nonlinear tolerance $2 \times 10^{-3}$ (blue) is now affected by noise, which is reduced by setting $M=5 \times 10^{5}$ and a tolerance of $7 \times 10^{-4}$ (red).

We choose parameters as in E3 of Table 1, with the exception of the slope of the sigmoid, $\alpha$, which is the continuation parameter. The computations are performed with $5 \times 10^{4}$ realizations, using a linear tolerance of $10^{-3}$, variance-reduced Jacobian-vector products with $\varepsilon=10^{-5}$, a relative nonlinear tolerance of $2 \times 10^{-3}$, and continuation steps $\Delta \alpha=0.14$. As we decrease $\alpha$, the stable front (labeled 1) loses stability at a symmetry-breaking bifurcation, giving rise to two partially locked-in states (labeled 3 and 4). These solutions correspond to those found via direct numerical simulations (see coarse profiles in Figure 4). As we increase $\alpha$, the stable front (labeled 1) becomes steeper: owing to our particular choice of $\mu(x)$, the limit of large $\alpha$ corresponds to two competing radical factions of the same size, and so in this limit the distribution of the average choice approaches a step function.

As expected, the number of realizations influences the continuation step size: in Figure 15 we recompute one of the branches in Figure 14 in the proximity of the symmetry-breaking bifurcation, with a much smaller continuation step, $\Delta \alpha=0.012$. The continuation with $M=5 \times 10^{4}$ and a relative nonlinear tolerance of $2 \times 10^{-3}$ (blue curve) is now affected by noise, which can be reduced by increasing the number of realizations to $5 \times 10^{5}$ and setting a tolerance of $7 \times 10^{-4}$ (red curve).

During continuation, we infer stability of a coarse solution $\boldsymbol{U}_{*}$ by computing eigenvalues of $\boldsymbol{D} \boldsymbol{F}\left(\boldsymbol{U}_{*}\right)=\boldsymbol{I}-\boldsymbol{D} \boldsymbol{\Phi}_{T}^{M}\left(\boldsymbol{U}_{*}\right)$. Since both $N$ and $M$ are relatively small, we form $\boldsymbol{D} \boldsymbol{F}\left(\boldsymbol{U}_{*}\right)$ using the finite difference approximation (4.11) $N$ times and then compute the full spectrum at once. For larger system sizes, matrix-free Arnoldi iterations can be employed to compute only the leading eigenvalues. In Figure 16 we plot the most unstable eigenvalue as a function of the bifurcation parameter, showing that the symmetry-breaking instability occurs at $\alpha \approx 1.5$. As a further remark on the accuracy of the variance-reduced Jacobian calculations, we plot the full spectrum for selected values of the continuation parameter, showing a clear separation between the leading real eigenvalue and a tight cluster of eigenvalues at the origin.

7. Conclusion. In this paper we have computed and continued in parameter space coarsegrained states for an ABM of consumer lock-in with heterogeneous agents. We first considered the simple case of homogeneous agents and found an explicit macroscopic evolution map for

Copyright (c) by SIAM. Unauthorized reproduction of this article is prohibited. 

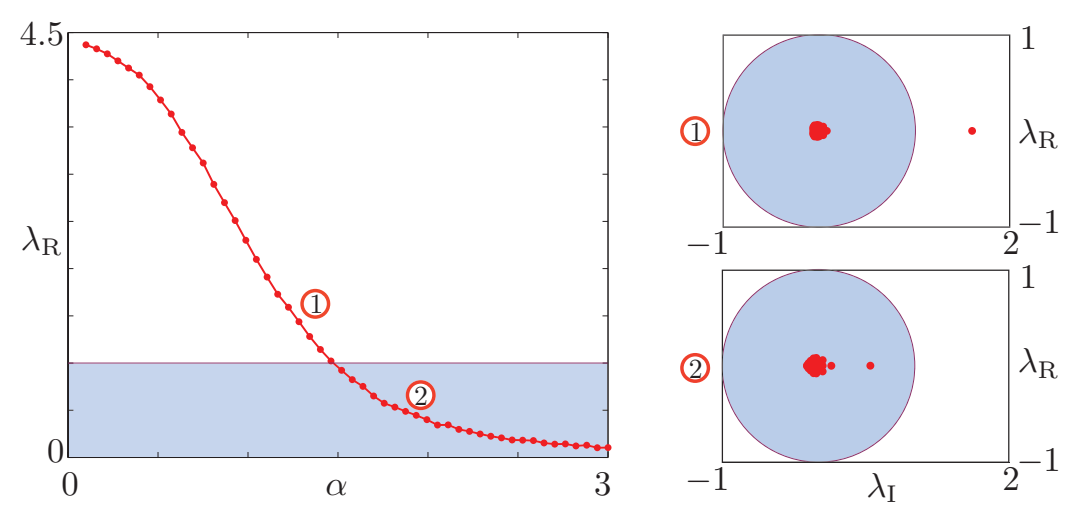

Figure 16. Eigenvalues of macroscopic front solutions on the symmetric branch of the bifurcation diagram in Figure 14. Left: The real part of the most unstable eigenvalue is plotted as a function of the continuation parameter $\alpha$. Right: Representative spectra along the branch.

the expectation of the mean purchase. As expected, this coarse description leads to a scenario similar to that found for linearly coupled oscillators subject to a double-well potential [5]: the first moment map stabilizes metastable locked-in states, which arise at a pitchfork bifurcation of the coarse map; introducing a homogeneous preference for one of the two products has the effect of breaking the pitchfork or, in the heat bath analogy, of introducing an asymmetric double-well potential.

The more interesting and challenging case of agents split into factions with opposite preferences leads to the formation of monotonically increasing macroscopic patterns, which have been computed using a large-dimensional coarse description. Our analysis reveals that, as the inhomogeneity becomes less pronounced, fronts undergo a symmetry-breaking instability. The resulting stable patterns are not fully locked in, since they feature pockets of resistance of each faction. An interesting future extension of this model could include a more granular modulation in the agents' preferences with nearest neighbor coupling: in this case the lock-in model would be posed on a spatially extended lattice and oscillatory one-dimensional inhomogeneities, or two-dimensional randomly distributed factions can be studied with the method presented here. In particular, the formulation of the two-dimensional coarse problem would follow the same steps as the one-dimensional case, even though it would naturally lead to a larger coarse system size. A further extension could include agent motility: in this way it would be possible to study how coherent spatiotemporal states, such as traveling fronts or bumps, are related to the spatial heterogeneity in agent preferences.

The core result of this paper is a strategy to evaluate variance-reduced Jacobian-vector products in equation-free methods. The main idea behind our approach is to exploit the nonuniqueness of the lifting operator to obtain a coarse time-stepper which depends smoothly on the coarse variables. In practice, this is achieved by using weighted averages in the restriction step and precomputing weights during the lifting step. We have shown that a direct consequence of using weights is that we gain full control over the linear solves, leading to wellbehaved GMRES iterations and, ultimately, to nonlinear convergence for large-dimensional coarse descriptions.

Copyright (c) by SIAM. Unauthorized reproduction of this article is prohibited. 
In order to assess the efficiency of the weights, we draw a comparison between weighted and unweighted Newton steps when the number of realizations $M$ is fixed. In the unweighted case, each Newton step requires one evaluation of $\Phi_{M}^{T}$ (one Bernoulli sampling, $M$ evolutions, one average) and then, for each GMRES step, a further evaluation of $\Phi_{M}^{T}$ involving $M$ further evolutions. In the weighted case, each Newton step requires one weighted evaluation of $\Phi_{M}^{T}$ (one Bernoulli sampling, $M$ evolutions, one manipulation of the constraint matrix, one linear solve, one weighted average) and then, for each GMRES step, one linear solve and one weighted average. Considering the improved GMRES and Newton-GMRES convergence, weighted operators seem more efficient, especially when running evolution steps is expensive.

We remark that, for the case under consideration, it was not possible to make a quantitative comparison of the efficiencies of weighted and unweighted coarse time-steppers, since the unweighted Newton-GMRES solver failed to converge for the inhomogeneous case. This reinforces the idea that, in large-dimensional coarse systems, noise can be harmful, and variancereduced Jacobian evaluations become an important ingredient in equation-free methods. Furthermore, weighted operators could be employed also in smaller coarse systems, such as those derived from Galerkin discretizations of spatially extended systems [26] or from chemical systems of moderate sizes [35].

A natural question arises as to whether weighted operators are applicable to other types of coarse-grained models. In section 4 we have presented weighted operators for the lock-in model, for which microscopic variables are binary numbers, but we envisage that similar ideas will be relevant in models where the microscopic variables are real numbers. In particular, it seems plausible to assume that the minimization problem (4.27)-(4.30) will remain valid if $\boldsymbol{u}^{m} \in \mathbb{R}^{N}$. Our current choice of the optimization problem for weights was driven by the following criteria:

1. The weighted realizations should satisfy the restriction exactly.

2. The weights should converge to 1 as $M \rightarrow \infty$.

3. The weights should depend continuously on the macroscopic state.

4. The weights should introduce minimal perturbations to the sampled probability distributions.

Other procedures to determine the weights are conceivable: we could allow only a limited number of weighting factors or use a different norm in the target function. When only allowing a limited number of weights, one clearly imposes additional artifacts on the represented probability distribution of realizations. Neither the resulting artifacts nor the effect of the choice of norm in the target function have been systematically studied in this work. These aspects, together with a more rigorous justification of weighted operators, will be the subject of future work.

Acknowledgments. We are grateful to Andrew Archer, Nigel Gilbert, David Lloyd, Alastair Rucklidge, Jan Sieber, and Anne Skeldon for stimulating discussions about this work.

\section{REFERENCES}

[1] G. Aletti, A. K. Naimzada, And G. NAldi, Mathematics and physics applications in sociodynamics simulation: The case of opinion formation and diffusion, in Mathematical Modeling of Collective Behavior in Socio-Economic and Life Sciences, Springer, New York, 2010, pp. 203-221.

Copyright (c) by SIAM. Unauthorized reproduction of this article is prohibited. 
[2] W. B. ARThur, Competing technologies, increasing returns, and lock-in by historical events, Econom. J., 99 (1989), pp. 116-131.

[3] W. B. Arthur, Positive feedbacks in the economy, Scientific American, 262 (1990), pp. 92-99.

[4] R. M. Axelrod, The Complexity of Cooperation: Agent-Based Models of Competition and Collaboration, Princeton University Press, Princeton, NJ, 1997.

[5] D. Barkley, I. G. Kevrekidis, and A. M. Stuart, The moment map: Nonlinear dynamics of density evolution via a few moments, SIAM J. Appl. Dyn. Syst., 5 (2006), pp. 403-434.

[6] D. J. Barnes And D. Chu, Introduction to Modeling for Biosciences, Springer-Verlag, New York, 2010.

[7] R. Borck, H. J. Кон, ANd M. Pflüger, Inefficient lock-in and subsidy competition, Internat. Econom. Rev., 53 (2012), pp. 1179-1204.

[8] R. E. Caflisch, Monte Carlo and quasi-Monte Carlo methods, Acta Numer., 7 (1998), pp. 1-49.

[9] E. Callen and D. Shapero, A theory of social imitation, Phys. Today, 27 (1974), p. 23.

[10] C. Castellano, S. Fortunato, and V. Loreto, Statistical physics of social dynamics, Rev. Modern Phys., 81 (2009), p. 591.

[11] B. K. Chakrabarti, A. Chakraborti, And A. Chatterjee, Econophysics and Sociophysics, John Wiley \& Sons, New York, 2007.

[12] B. A. Cipra, An introduction to the Ising model, Amer. Math. Monthly, 94 (1987), pp. 937-959.

[13] J. Cisternas, C. W. Gear, S. Levin, And I. G. Kevrekidis, Equation-free modelling of evolving diseases: Coarse-grained computations with individual-based models, Proc. R. Soc. Lond. Ser. A Math. Phys. Eng. Sci., 460 (2004), pp. 2761-2779.

[14] O. Corradi, P. G. HJorth, And J. Starke, Equation-free detection and continuation of a Hopf bifurcation point in a particle model of pedestrian flow, SIAM J. Appl. Dyn. Syst., 11 (2012), pp. 1007-1032.

[15] P. A. DAvid, Clio and the economics of QWERTY, Amer. Econom. Rev., 75 (1985), pp. 332-337.

[16] B. Düring, P. Markowich, J. Pietschmann, and M. Wolfram, Boltzmann and Fokker-Planck equations modelling opinion formation in the presence of strong leaders, Proc. R. Soc. Ser. A Math. Phys. Eng. Sci., 465 (2009), pp. 3687-3708.

[17] W. E. And B. Engquist, The heterogeneous multi-scale methods, Commun. Math. Sci., 1 (2003), pp. 87132.

[18] J. M. Epstein, Learning to be thoughtless: Social norms and individual computation, Comput. Econom., 18 (2001), pp. 9-24.

[19] J. M. Epstein, Modeling civil violence: An agent-based computational approach, Proc. Natl. Acad. Sci. USA, 99 (2002), p. 7243.

[20] J. M. Epstein And R. Axtell, Growing Artificial Societies: Social Science from the Bottom Up, The MIT Press, Cambridge, MA, 1996.

[21] R. Erban, T. A. Frewen, X. Wang, T. C. Elston, R. Coifman, B. Nadler, and I. G. Kevrekidis, Variable-free exploration of stochastic models: A gene regulatory network example, J. Chem. Phys., 126 (2007), 155103.

[22] R. Erban, I. G. Kevrekidis, and H. G. Othmer, An equation-free computational approach for extracting population-level behavior from individual-based models of biological dispersal, Phys. D, 215 (2006), pp. 1-24.

[23] S. Galam, Y. Gefen, And Y. Shapir, Sociophysics: A new approach of sociological collective behaviour. I. Mean-behaviour description of a strike, J. Math. Sociology, 9 (1982), pp. 1-13.

[24] M. Garlick And M. Chli, An agent-based simulation of lock-in dynamics in a duopoly, in Proceedings of the 9th International Conference on Autonomous Agents and Multiagent Systems, Toronto, Canada, 2010, pp. 1545-1546.

[25] L. Gauvin, J. Vannimenus, and J. P. Nadal, Phase diagram of a Schelling segregation model, Eur. Phys. J. B, 70 (2009), pp. 293-304.

[26] C. W. Gear, I. G. Kevrekidis, and C. Theodoropoulos, 'Coarse' integration/bifurcation analysis via microscopic simulators: Micro-Galerkin methods, Computers Chem. Engrg., 26 (2002), pp. 941-963.

[27] G. N. Gilbert, Agent-Based Models, Vol. 153, Sage Publications, New York, 2008.

[28] J. A. Glazier And F. Graner, Simulation of the differential adhesion driven rearrangement of biological cells, Phys. Rev. E, 47 (1993), pp. 2128-2154.

[29] N. I. M. Gould, M. E. Hribar, And J. Nocedal, On the solution of equality constrained quadratic programming problems arising in optimization, SIAM J. Sci. Comput., 23 (2001), pp. 1376-1395.

Copyright (C) by SIAM. Unauthorized reproduction of this article is prohibited. 
[30] F. Graner And J. A. Glazier, Simulation of biological cell sorting using a two-dimensional extended Potts model, Phys. Rev. Lett., 69 (1992), pp. 2013-2016.

[31] R. Hegselmann and U. Krause, Opinion dynamics and bounded confidence models, analysis, and simulation, J. Artificial Societies Social Simul., 5 (2002); available online from http://jasss.soc.surrey. ac.uk $/ 5 / 3 / 2 / 2 . p d f$.

[32] D. Helbing, Quantitative Sociodynamics: Stochastic Methods and Models of Social Interaction Processes, Springer-Verlag, New York, 2011.

[33] D. Helbing, I. Farkas, And T. Vicsek, Simulating dynamical features of escape panic, Nature, 407 (2000), pp. 487-490.

[34] R. A. Holley and T. M. Liggett, Ergodic theorems for weakly interacting infinite systems and the voter model, Ann. Probab., 3 (1975), pp. 643-663.

[35] R. B. Hoyle, D. Avitabile, And A. M. Kierzek, Equation-free analysis of two-component system signalling model reveals the emergence of co-existing phenotypes in the absence of multistationarity, PLoS Comput. Biol., 8 (2012), e1002396.

[36] M. JANSSEN AND W. JAGER, An integrated approach to simulating behavioural processes: A case study of the lock-in of consumption patterns, J. Artificial Societies Social Simul., 2 (1999), pp. 21-35.

[37] I. G. Kevrekidis, C. W. Gear, J. M. Hyman, P. G. Kevrekidis, O. Runborg, and C. TheodorOPOULOS, Equation-free, coarse-grained multiscale computation: Enabling microscopic simulators to perform system-level tasks, Commun. Math. Sci., 1 (2003), pp. 715-762.

[38] I. G. Kevrekidis And G. Samaey, Equation-free multiscale computation: Algorithms and applications, Ann. Rev. Phys. Chem., 60 (2009), pp. 321-344.

[39] A. Kolpas, J. Moehlis, T. A Frewen, and I. G. Kevrekidis, Coarse analysis of collective motion with different communication mechanisms, Math. Biosci., 214 (2008), pp. 49-57.

[40] A. Kolpas, J. Moehlis, And I. G. Kevrekidis, Coarse-grained analysis of stochasticity-induced switching between collective motion states, Proc. Natl. Acad. Sci. USA, 104 (2007), pp. 5931-5935.

[41] C. R. LAInG, On the application of equation-free modelling to neural systems, J. Comput. Neurosci., 20 (2006), pp. 5-23.

[42] C. R. Laing, T. Frewen, and I. G. Kevrekidis, Reduced models for binocular rivalry, J. Comput. Neurosci., 28 (2010), pp. 459-476.

[43] M. Lewenstein, Statistical mechanics of social impact, Phys. Rev. A, 45 (1992), pp. 763-776.

[44] J. Lorenz, Continuous opinion dynamics under bounded confidence: A survey., Internat. J. Modern Phys. C, 18 (2007), pp. 1819-1838.

[45] J. LorenZ, Fostering consensus in multidimensional continuous opinion dynamics under bounded confidence, in Managing Complexity: Insights, Concepts, Applications, Springer, New York, 2008, pp. 321334.

[46] K. Madsen, H. Nielsen, and O. Tingleff, Optimization with constraints, Technical report, Department of Informatics and Mathematical Modelling (IMM), Technical University of Denmark, Kongens Lyngby, Denmark, http://www.orbit.dtu.dk/services/downloadRegister/2721110/imm4213.pdf, 2004.

[47] C. Marschler, J. Sieber, R. Berkemer, A. Kawamoto, and J. Starke, Implicit Methods for Equation-Free Analysis: Convergence Results and Analysis of Emergent Waves in Microscopic Traffic Models, Tech. rep., http://arxiv.org/abs/1301.6044, 2013.

[48] M. Marsili, F. Vega-Redondo, and F. Slanina, The rise and fall of a networked society: A formal model, Proc. Natl. Acad. Sci. USA, 101 (2004), pp. 1439-1442.

[49] M. Moussaïd, N. Perozo, S. Garnier, D. Helbing, and G. Theraulaz, The walking behaviour of pedestrian social groups and its impact on crowd dynamics, PLoS One, 5 (2010), e10047.

[50] C. Nikolai and G. Madey, Tools of the trade: A survey of various agent based modeling platforms, J. Artificial Societies Social Simul., 12 (2009), p. 2.

[51] O. Runborg, C. Theodoropoulos, and I. G. Kevrekidis, Effective bifurcation analysis: A timestepper-based approach, Nonlinearity, 15 (2002), pp. 491-511.

[52] T. C. Schelling, Micromotives and Macrobehavior, W.W. Norton \& Company, New York, 2006.

[53] F. Schweitzer, Modeling Complexity in Economic and Social Systems, World Scientific, Singapore, 2002.

[54] M. B. Short, A. L. Bertozzi, and P. J. Brantingham, Nonlinear patterns in urban crime: Hotspots, bifurcations, and suppression, SIAM J. Appl. Dyn. Syst., 9 (2010), pp. 462-483.

Copyright (C) by SIAM. Unauthorized reproduction of this article is prohibited. 
[55] K. G. Spiliotis, L. Russo, And C. I. Sietros, Symmetry and symmetry-breaking of the emergent dynamics of the discrete stochastic majority-voter model, Ann. Rev. Chaos Theory Bifur. Dynam. Syst., 2 (2012), pp. 1-20.

[56] K. G. Spiliotis And C. I. Siettos, A timestepper-based approach for the coarse-grained analysis of microscopic neuronal simulators on networks: Bifurcation and rare-events micro-to macro-computations, Neurocomputing, 74 (2011), pp. 3576-3589.

[57] H. E. Stanley, Introduction to Phase Transitions and Critical Phenomena, Vol. 1, Oxford University Press, Oxford, UK, 1987.

[58] D. Stauffer and S. Solomon, Ising, Schelling and self-organising segregation, Eur. Phys. J. B, 57 (2007), pp. 473-479.

[59] K. Sznajd-Weron And J. Sznajd, Opinion evolution in closed community, Internat. J. Modern Phys. C, 11 (2000), pp. 1157-1165.

[60] K. Sznajd-Weron and J. Sznajd, Who is left, who is right?, Phys. A, 351 (2005), pp. 593-604.

[61] K. Sznajd-Weron, M. Tabiszewski, and A. M. Timpanaro, Phase transition in the Sznajd model with independence, Europhys. Lett. EPL, 96 (2011), p. 48002.

[62] A. Troisi, V. Wong, And M. A. Ratner, An agent-based approach for modeling molecular selforganization, Proc. Natl. Acad. Sci. USA, 102 (2005), pp. 255-260.

[63] A. C. Tsoumanis, C. I. Siettos, G. V. Bafas, And I. G. Kevrekidis, Equation-free multiscale computations in social networks: From agent-based modeling to coarse-grained stability and bifurcation analysis, Internat. J. Bifur. Chaos Appl. Sci. Engrg., 20 (2010), pp. 3673-3688.

[64] D. J. Watts and S. H. Strogatz, Collective dynamics of small-world networks, Nature, 393 (1998), pp. $440-442$.

[65] W. Weidlich, The statistical description of polarization phenomena in society, British J. Math. Statist. Psych., 24 (1971), pp. 251-266.

[66] W. Weidlich, Sociodynamics: A Systematic Approach to Mathematical Modelling in the Social Sciences, Harwood Academic, Reading, UK, 2000.

[67] W. E. Weinan, B. Engquist, X. Li, W. Ren, and E. Vanden-Eijnden, Heterogeneous multiscale methods: A review, Commun. Comput. Phys., 2 (2007), pp. 367-450.

Copyright $@$ by SIAM. Unauthorized reproduction of this article is prohibited. 